\title{
Strong bulk photovoltaic effect and second-harmonic generation in two-dimensional selenium and tellurium
}

\author{
Meijuan Cheng, ${ }^{1}$ Zi-Zhong Zhu, ${ }^{1,2, *}$ and Guang-Yu Guo ${ }^{3,4, \oplus}$ \\ ${ }^{1}$ Department of Physics, Collaborative Innovation Center for Optoelectronic Semiconductors and Efficient Devices, \\ Key Laboratory of Low Dimensional Condensed Matter Physics (Department of Education of Fujian Province), \\ Jiujiang Research Institute, Xiamen University, Xiamen 361005, China \\ ${ }^{2}$ Fujian Provincial Key Laboratory of Theoretical and Computational Chemistry, Xiamen 361005, China \\ ${ }^{3}$ Department of Physics and Center for Theoretical Physics, \\ National Taiwan University, Taipei 10617, Taiwan \\ ${ }^{4}$ Physics Division, National Center for Theoretical Sciences, Taipei 10617, Taiwan
}

(Dated: June 21, 2021)

\begin{abstract}
Few-layer selenium and tellurium films have been recently prepared, and they provide a new platform to explore novel properties of two-dimensional (2D) elemental materials. In this work, we have performed a systematic first-principles study on the electronic, linear and nonlinear optical (NLO) properties of atomically thin selenium and tellurium films within the density-functional theory with the generalized gradient approximation plus scissors correction using the band gaps from the relativistic hybrid Heyd-Scuseria-Erzerhof functional calculations. The underlying atomic structures of these materials are determined theoretically using the SCAN exchange-correlation functional. Interestingly, we find that few-layer Se and Te possess large second-harmonic generation (SHG), linear electro-optic (LEO) effect and bulk photovoltaic effect. In particular, trilayer (TL) Te possesses large SHG coefficient, being more than 65 times larger than that of GaN, a widely used NLO material. Bilayer (BL) Te has huge static SHG coefficient $\chi_{x y y}^{(2)}(0)$, being more than 100 times larger than that of GaN. Furthermore, monolayer (ML) Se possesses large SHG coefficient with $\chi_{x y y}^{(2)}$ being six times larger than that of GaN. Both ML Se and BL Te possess large linear electro-optic coefficients $r_{x y y}(0)$ and $r_{y z x}(0)$, which is about 6 times and 5 times larger than that of bulk GaN polytypes, respectively. Moreover, we predict that TL Te exhibits strong bulk photovoltaic effect (BPVE) with shift current conductivity of $\sim 440 \mu \mathrm{A} / \mathrm{V}^{2}$, being greater than that of GeS, a polar system with the largest BPVE found so far. Although the shift current conductivities of bulk and 2D Se are comparable, the shift current conductivities of TL Te are five times larger than that of bulk Te. Finally, an analysis of the calculated electronic band structures indicates that the strong NLO responses of $2 \mathrm{D}$ Se and Te materials are primarily derived from their low-dimensional structures with high anisotropy, directional covalent bonding, lone-pair electrons and relatively small band gaps. These findings provide a practical strategy to search for excellent NLO and BPVE materials.
\end{abstract}

\section{INTRODUCTION}

Noncentrosymmetric materials under intense optical fields may generate large even-order nonlinear optical (NLO) responses such as second-order NLO susceptibility $\left(\chi^{(2)}\right)$ [1, 2]. Large second-order NLO susceptibility is of great importance for many technological applications such as electro-optical switches, light signal modulators and frequency conversion. As one of the best-known second-order NLO optical responses [2], the second-harmonic generation ( $\mathrm{SHG}$ ) has been widely used as surface probes and frequency doublers [1]. Since the 1960s, the SHG has been investigated extensively in bulk semiconductors [2 8] and more recently also in one-dimensional (see, e.g., Refs. [9, 10] and references therein) and two-dimensional (see, e.g., Refs. [11 18] and references therein) materials. Linear electro-optic (LEO) effect, another second-order NLO response of a noncentrosymmetric material, refers to the linear refractive in-

\footnotetext{
* zzhu@xmu.edu.cn

$\dagger$ gyguo@phys.ntu.edu.tw
}

dex change $(\Delta n)$ with the applied electric field strength $(E), \Delta n=n^{3} r E / 2$, where $n$ is the refraction index and $r$ is the LEO coefficient [2]. The LEO effect thus allows one to use an electrical signal to control the amplitude, phase or direction of a light beam in the NLO material, and leads to a widely used means for high-speed optical modulation and sensing devices (see, e.g., Ref. [19] and references therein).

In recent years we have seen a surge of interest in bulk photovoltaic effect (BPVE), another nonlinear optical response, referring to the generation of photovoltage or photocurrent in noncentrosymmetric materials. Earlier studies in 1960s demonstrated intrinsic photocurrents in ferroelectric oxide $\mathrm{BaTiO}_{3}$ [20]. Afterwards, the BPVE was reported in $\mathrm{LiNbO}_{3}$ [21]. Recently, Young et al. demonstrated theoretically that shift photocurrent dominates the BPVE in $\mathrm{BaTiO}_{3}$. 22] Then, Bhatnagar et al. experimentally discovered large open-circuit photovoltages and thus proved the BPVE in multiferroic $\mathrm{BiFeO}_{3}$ (BFO) thin films. 23] After this, Brehm et al. calculated the shift current conductivity for polar compounds $\mathrm{LiAsS}_{2}, \mathrm{LiAsSe}_{2}$ and $\mathrm{NaAsSe}_{2}$, and found that they exhibit shift current which is nearly 20 times larger than that of $\mathrm{BiFeO}_{3}$ [24]. Recently, Rangel et al. theoreti- 
cally investigated the BPVE in single-layer monochalcogenides [25]. More recently, Gong et al. found that ferroelectric semiconductor GeTe possesses large shift current response due to narrow band gap and high covalency [26].

Selenium and tellurium bulks [27 31] and chains [3240] have been investigated extensively due to their unique properties. Nevertheless, their two-dimensional (2D) counterparts have not been fabricated and investigated almost untill the present day. In 2017, Qin et al. first achieved the controlled growth of a large-size 2D selenium nanosheet using a physical vapor deposition method [4]. Zhu et al. revealed that the metalloid element Te exists three phases of $2 \mathrm{D}$ monolayer, $1 \mathrm{~T}-\mathrm{MoS}_{2}$-like $(\alpha$ $\mathrm{Te})$ structure, tetragonal $(\beta$-Te $)$ and $2 \mathrm{H}-\mathrm{MoS}_{2}$-like $(\gamma$ Te) structures, named tellurene [42]. In particular, $\beta$ Te has been synthesized on highly oriented pyrolytic graphite (HOPG) substrates by using molecular beam epitaxy [42]. Since then, extensive investigations on 2D Se and Te materials have been conducted both theoretically [43 49] and experimentally [50 55]. Notably, Huang et al. recently grew monolayer (ML) and few-layer Te films on a graphene/6H-SiC(0001) substrate by molecular beam epitaxy. The Te films consist of the parallel helical chains exposing the Te bulk $b-c$ (or $a-c$ ) facet [56]. Remarkably, these ML and few-layer films are noncentrosymmetric and thus are potentially excellent $2 \mathrm{D}$ second-order NLO materials. Soon afterwards, several theoretical groups investigated the structural, electronic, optical properties [57], ferroelectricity and spintextures [58], as well as phase transitions [59] in fewlayer tellurium films. Strong optical absorption, high carrier mobility, outstanding environmental stability, intrinsic anisotropy, layer-dependent and almost direct band gap of these films have been observed, indicating their promising applications in electronics, optoelectronics, vapor sensors, spintronics, biomedicine.

Stimulated by these experimental breakthroughs, here we have carried out a systematic first-principles investigation on the SHG and BPVE in these $2 \mathrm{D}$ selenium and tellurium based on the density functional theory calculations. This paper is organized as follows. In Sec. II, we present the theoretical approach and computational details. In Sec. III, we report the electronic band structures of 2D $\alpha$-phase Se and Te. In Sec. IV, we present the real and imaginary parts of the optical dielectric function. In Sec. V, the calculated LEO and SHG coefficients over the entire optical frequency range are presented. Furthermore, the second-order optical susceptibility is analyzed in terms of one- and two-photon resonances. In Sec. VI, we present the calculated shift current conductivities. Comparison of the obtained results of 2D Se and Te with other known NLO materials suggests that they have promising applications in NLO devices such as novel solar-cell, photodetector, SHG, electro-optical modulator and electric optical switches. In Sec. VII, we discuss the possible origins of the strong NLO responses of 2D Se and Te in terms of the calculated deformation charge density distribution. Finally, a summary is given in Sec. VIII.

\section{STRUCTURE AND COMPUTATIONAL METHOD}

The $\alpha$-phase crystalline structure of bulk selenium and tellurium is schematically shown in Figs. 1(a), 1(b) and 1(c). It consists of parallel-aligned helical chains arranged in a hexagonal array [60, 61]. Every atom is covalently bonded to two neighboring atoms along each chain, and interacts weakly with atoms on the adjacent chains. Figure 1(a) shows that bulk selenium and tellurium may be viewed as a layered structure. Different layers are stacked in the direction perpendicular to $a-c($ or $b-c)$ planes and each layer consists of one-dimensional array of single helical chains stretched to infinity along the $c$-axis. An $n$ layer 2D structure is made of $n$ helical chain layers stacked in the bulk mode [see Figs. 1(d), 1(e) and 1(f)]. Interestingly, the space group of an odd-number layer structure is $P 2\left(C_{2}^{1}\right)$, while that of an even-number layer structure is $P 2_{1}\left(C_{2}^{2}\right)$ (see Table II). Note that $\alpha$-phase bulk and few-layer structures all possess broken inversion symmetry, the necessary condition for nonzero secondorder NLO responses. However, the $\alpha$-phase ML tellurium is unstable against the $\beta$-phase, a structure with centrosymmetry [see Figs. 1(g), 1(h) and 1(i)]. Nonetheless, for all other atomically thin Te films, $\alpha$-phase is the most stable structure. Also, for all the atomically thin Se films, $\alpha$-phase is the most stable structure. Therefore, we consider only bilayer (BL) and trilayer (TL) tellurium as well as ML, BL and TL selenium. In the present calculations, 2D selenium and tellurium structures are modeled by using the slab-superlattice approach. The separations between the $2 \mathrm{D}$ structures adopted are about $20 \AA$, to ensure negligible interaction between the periodic images.

TABLE I. Theoretical lattice constants (in the unit of $\AA$ ) of bulk selenium and tellurium calculated with the SCAN, optB88-vdW, DFT-D2 and PBE functionals, compared with the experimental lattice constants (exp).

\begin{tabular}{|c|c|c|c|c|c|c|}
\hline & & SCAN & optB88-vdW & PBE-D2 & $\mathrm{PBE}$ & $\exp$ \\
\hline \multirow[t]{2}{*}{ Se } & $a$ & 4.4336 & 4.2348 & 4.2517 & 4.4984 & $4.3662^{\mathrm{a}}$ \\
\hline & $c$ & 4.9984 & 5.1319 & 5.1065 & 5.0526 & $4.9536^{\mathrm{a}}$ \\
\hline \multirow[t]{2}{*}{$\mathrm{Te}$} & $a$ & 4.4578 & 4.4816 & 4.3357 & 4.5085 & $4.4511^{\mathrm{b}}$ \\
\hline & $c$ & 5.9291 & 5.9951 & 6.0300 & 5.9593 & $5.9262^{\mathrm{b}}$ \\
\hline
\end{tabular}

Our density functional theory calculations are performed by using the highly accurate projector augmented wave (PAW) method [62], as implemented in the VASP package [63, 64]. A large plane-wave cutoff energy of $450 \mathrm{eV}$ is used throughout. The PAW potentials for Se $4 s^{2} 4 p^{4}$ and for Te $5 s^{2} 5 p^{4}$ are adopted to describe the electron-ion interaction. The theoretical atomic positions and lattice constants are obtained when the forces acting on all the atoms are less than $0.001 \mathrm{eV} / \AA$ and the stresses are less $1.0 \mathrm{kBar}$. The total energy convergence 


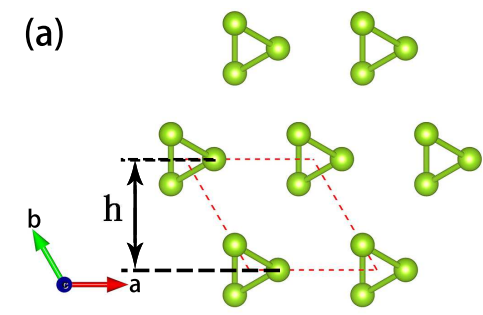

(b)

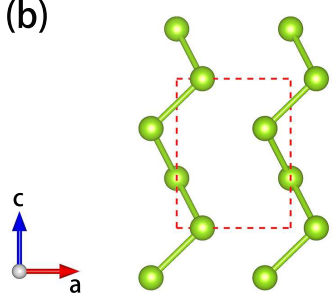

(c)

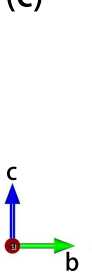

(d)

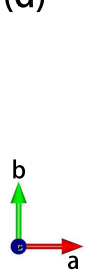

(e)
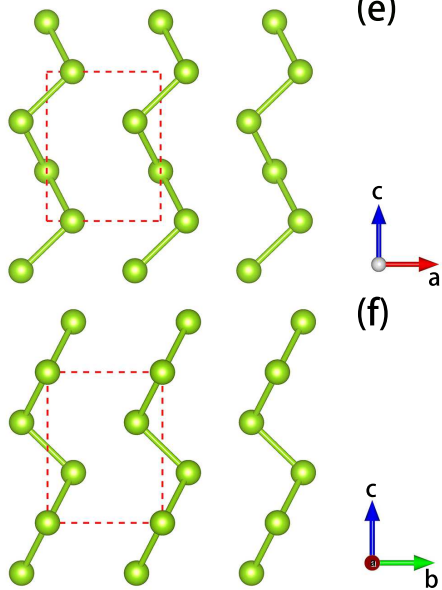

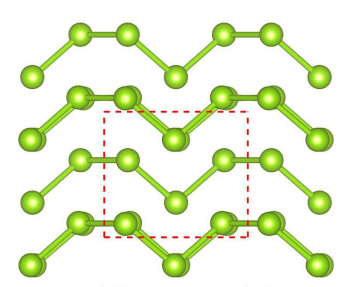

(g)

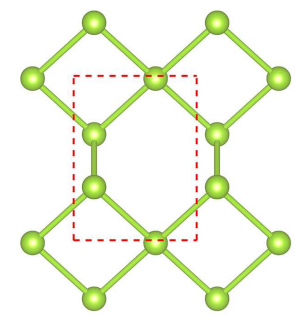

(h)
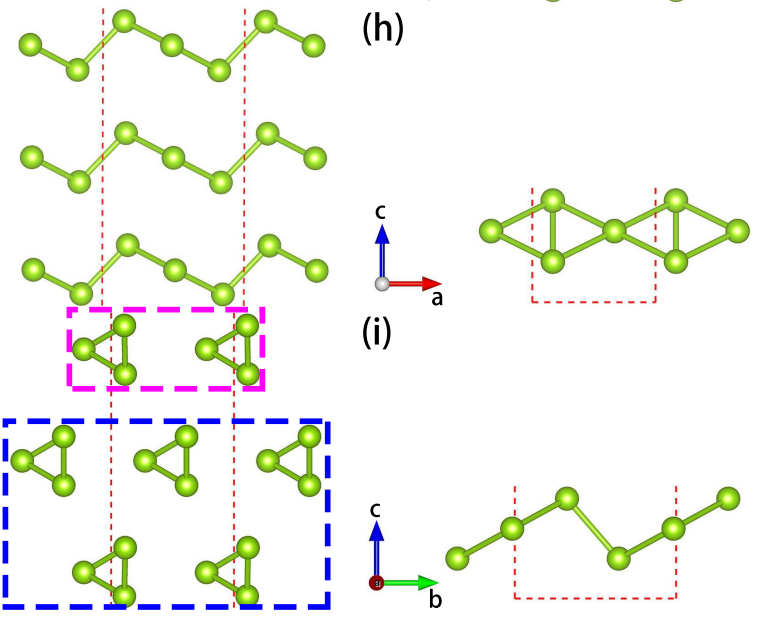

(i)

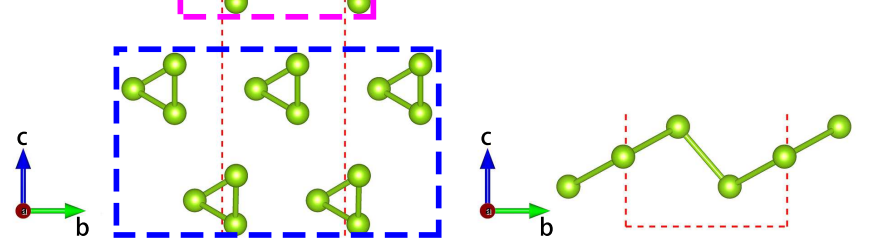

FIG. 1. (a) Top and (b,c) side views of the $\alpha$-phase crystalline structure of bulk selenium and tellurium. (d) Top and (e, f) side views of the $\alpha$-phase TL structure. In (a), symbol $h$ denotes the effective ML thickness. In (f), the blue dashed rectangle indicates the $\alpha$-phase BL structure. The magenta dashed rectangle indicates the $\alpha$-phase ML structure. (g) Top and (h, i) side views of the $\beta$-phase ML Te. The red dashed lines indicate the unit cell of each structure.

TABLE II. Space group, calculated lattice constants $(a, b$ and $c)$, effective thickness $(h)$, layer-averaged interchain $(R)$ and intrachain $(r)$ distances as well as their ratio $(R / r)$ of bulk and 2D selenium and tellurium obtained using the SCAN functional.

\begin{tabular}{|c|c|c|c|c|c|c|c|}
\hline & bulk Se & ML Se & $\mathrm{BL} \mathrm{Se}$ & TL Se & bulk Te & $\mathrm{BL} \mathrm{Te}$ & TL Te \\
\hline space group & $P 3_{1} 21$ & $P 2$ & $P 2_{1}$ & $P 2$ & $P 3_{1} 21$ & $P 2_{1}$ & $P 2$ \\
\hline$a(\AA)$ & 4.434 & 4.918 & 4.955 & 4.968 & 4.458 & 5.791 & 5.839 \\
\hline$b(\AA)$ & 4.434 & 4.198 & 4.287 & 4.336 & 4.458 & 4.311 & 4.367 \\
\hline$c(\AA)$ & 4.998 & & & & 5.929 & & \\
\hline$h(\AA)$ & & 3.781 & 7.562 & 11.344 & & 7.71 & 11.564 \\
\hline$R(\AA)$ & 3.50 & 3.34 & 3.55 & 3.54 & 3.47 & 3.51 & 3.48 \\
\hline$r(\AA)$ & 2.38 & 2.36 & 2.37 & 2.37 & 3.17 & 2.85 & 2.84 \\
\hline$R / r$ & 1.47 & 1.42 & 1.50 & 1.49 & 1.09 & 1.23 & 1.23 \\
\hline
\end{tabular}

criterion for the self-consistent electronic structure calculations is $10^{-6} \mathrm{eV}$. The accurate tetrahedron method [65] is used for the Brillouin zone integration. The selfconsistent charge density calculations are performed with $k$-point meshes of $18 \times 20 \times 1$ for $2 \mathrm{D}$ Se and $15 \times 20 \times 1$ for multilayer Te.

To obtain accurate structure parameters, we first perform structural optimizations for bulk selenium and tellurium with the conjugate gradient technique using the SCAN [66, 67], optB88-vdW 68], Perdew-BurkeErnzerhof (PBE) 69], DFT-D2 [70] exchange-correlation functionals. The calculated lattice constants are listed in Table I. The lattice constants calculated using the SCAN functional are in the best agreement with experiments
60, 61] (see Table I). Therefore, in all the geometric optimization calculations for the 2D structures, we adopt the SCAN functional.

Using the structural parameters for the 2D structures determined by using the SCAN functional, we first perform the self-consistent band structure calculations within the generalized gradient approximation (GGA) of the PBE functional [69]. We then calculate the optical dielectric function and NLO responses of the 2D structures from the calculated band structures within the linear response formalism with the independent-particle approximation. In particular, the imaginary part of the dielectric function $\varepsilon(\omega)$ due to direct interband transition in 
the atomic units is given by (see, e.g., Refs. [9, 71]),

$$
\varepsilon_{a}^{\prime \prime}(\omega)=\frac{4 \pi^{2}}{\Omega \omega^{2}} \sum_{i \in V B, j \in C B} \sum_{\mathbf{k}} w_{\mathbf{k}}\left|p_{i j}^{a}\right|^{2} \delta\left(\epsilon_{\mathbf{k} j}-\epsilon_{\mathbf{k} i}-\omega\right),
$$

where $\Omega$ is the unit-cell volume, $w_{\mathbf{k}}$ is the weight for the $\mathbf{k}$ point and $\omega$ is the photon energy. $\mathrm{VB}$ and $\mathrm{CB}$ stand for the valence and conduction bands, respectively. The dipole transition matrix elements $p_{i j}^{a}=\left\langle\mathbf{k} j\left|\hat{p}_{a}\right| \mathbf{k} i\right\rangle$, where $\hat{p}_{a}$ represents Cartesian component $a$ of the dipole operator, are obtained from the band structures within the PAW formalism [72]. Here $|\mathbf{k} n\rangle$ is the $n$th Bloch state function with momentum $\mathbf{k}$. The real part of the dielectric function is then obtained from the calculated $\varepsilon^{\prime \prime}(\omega)$ by the Kramer-Kronig transformation [9, 71],

$$
\varepsilon^{\prime}(\omega)=1+\frac{2}{\pi} \mathbf{P} \int_{0}^{\infty} d \omega^{\prime} \frac{\omega^{\prime} \varepsilon^{\prime \prime}\left(\omega^{\prime}\right)}{\omega^{\prime 2}-\omega^{2}},
$$

where $\mathbf{P}$ denotes the principal value of the integral.

The imaginary part of the second-order NLO susceptibility due to direct interband transitions is given by 9, 10

$\chi_{a b c}^{\prime \prime(2)}(-2 \omega, \omega, \omega)=\chi_{a b c, V E}^{\prime \prime(2)}(-2 \omega, \omega, \omega)+\chi_{a b c, V H}^{\prime \prime(2)}(-2 \omega, \omega, \omega)$,

where the contribution due to the so-called virtualelectron (VE) process is [9, 10]

$$
\begin{gathered}
\chi_{a b c, V E}^{\prime \prime(2)}=-\frac{\pi}{2 \Omega} \sum_{i \in V B} \sum_{j, l \in C B} \sum_{\mathbf{k}} w_{\mathbf{k}}\left\{\frac{\operatorname{Im}\left[p_{j l}^{a}\left\langle p_{l i}^{b} p_{i j}^{c}\right\rangle\right]}{\epsilon_{l i}^{3}\left(\epsilon_{l i}+\epsilon_{j i}\right)} \delta\left(\epsilon_{l i}-\omega\right)\right. \\
\left.-\frac{\operatorname{Im}\left[p_{i j}^{a}\left\langle p_{j l}^{b} p_{l i}^{c}\right\rangle\right]}{\epsilon_{l i}^{3}\left(2 \epsilon_{l i}-\epsilon_{j i}\right)} \delta\left(\epsilon_{l i}-\omega\right)+\frac{16 \operatorname{Im}\left[p_{i j}^{a}\left\langle p_{j l}^{b} p_{l i}^{c}\right\rangle\right]}{\epsilon_{j i}^{3}\left(2 \epsilon_{l i}^{3}-\epsilon_{j i}^{3}\right)} \delta\left(\epsilon_{j i}-2 \omega\right)\right\},
\end{gathered}
$$

and that due to the virtual-hole $(\mathrm{VH})$ process is $[9,10$

$$
\begin{aligned}
& \chi_{a b c, V H}^{\prime \prime(2)}=\frac{\pi}{2 \Omega} \sum_{i, l \in V B} \sum_{j \in C B} \sum_{\mathbf{k}} w_{\mathbf{k}}\left\{\frac{\operatorname{Im}\left[p_{l i}^{a}\left\langle p_{i j}^{b} p_{j l}^{c}\right\rangle\right]}{\epsilon_{j l}^{3}\left(\epsilon_{j l}+\epsilon_{j i}\right)} \delta\left(\epsilon_{j l}-\omega\right)\right. \\
& \left.-\frac{\operatorname{Im}\left[p_{i j}^{a}\left\langle p_{j l}^{b} p_{l i}^{c}\right\rangle\right]}{\epsilon_{j l}^{3}\left(2 \epsilon_{j l}-\epsilon_{j i}\right)} \delta\left(\epsilon_{j l}-\omega\right)+\frac{16 \operatorname{Im}\left[p_{i j}^{a}\left\langle p_{j l}^{b} p_{l i}^{c}\right\rangle\right]}{\epsilon_{j i}^{3}\left(2 \epsilon_{j l}-\epsilon_{j i}\right)} \delta\left(\epsilon_{j i}-2 \omega\right)\right\} .
\end{aligned}
$$

Here $\epsilon_{j i}=\epsilon_{\mathbf{k} j}-\epsilon_{\mathbf{k} i}$ and $\left\langle p_{j l}^{b} p_{l i}^{c}\right\rangle=\frac{1}{2}\left(p_{j l}^{b} p_{l i}^{c}+p_{l i}^{b} p_{j l}^{c}\right)$. The real part of the second-order NLO susceptibility is obtained from the calculated $\chi_{a b c}^{\prime \prime(2)}$ by the Kramer-Kronig transformation [9, 10]

$$
\chi^{\prime(2)}(-2 \omega, \omega, \omega)=\frac{2}{\pi} \mathbf{P} \int_{0}^{\infty} d \omega^{\prime} \frac{\omega^{\prime} \chi^{\prime \prime(2)}\left(2 \omega^{\prime}, \omega^{\prime}, \omega^{\prime}\right)}{\omega^{\prime 2}-\omega^{2}} .
$$

The LEO coefficient $r_{a b c}(\omega)$ is related to the second-order NLO susceptibility $\chi_{a b c}^{(2)}(-\omega, \omega, 0)[\underline{5}, 53]$. In the zero frequency limit [5, 73],

$$
r_{a b c}(0)=-\frac{2}{\varepsilon_{a}(0) \varepsilon_{b}(0)} \lim _{\omega \rightarrow 0} \chi_{a b c}^{(2)}(-2 \omega, \omega, \omega) .
$$

In the very low frequency region, i.e. the photon energy $\hbar \omega$ well below the band gap, $\chi_{a b c}^{(2)}(-2 \omega, \omega, \omega)$ and $n(\omega)$ are nearly constant. In this case, the LEO coefficient $r_{a b c}(\omega) \approx r_{a b c}(0)[9,13]$.

The DC shift current along the $a$-axis is given by

$$
J_{a}=\sum_{b c} \sigma_{a b c}(0 ; \omega,-\omega) E_{b}(\omega) E_{c}(-\omega),
$$

where $\sigma_{a b c}(0 ; \omega,-\omega)$ is the third-rank conductivity tensor. [74] Within the length gauge formalism, $\sigma_{a b c}(0 ; \omega,-\omega)$ can be written in terms of the interband position matrix element $r_{i j}^{a}$ and its momentum derivative $r_{i j ; b}^{a}$ (see Eq. (57) in Ref. [74]). By replacing $r_{i j}^{a}$ with $p_{i j}^{a} / i \epsilon_{i j}$, one would obtain

$$
\begin{array}{r}
\sigma_{a b c}(0 ; \omega,-\omega)=\frac{\pi}{\Omega} \sum_{i \in V B} \sum_{j \in C B} \frac{1}{\epsilon_{i j}^{2}} \sum_{\mathbf{k}} w_{\mathbf{k}} \\
\sum_{l \neq i, j} \operatorname{Im}\left\{\frac{p_{i l}^{a}\left\langle p_{j i}^{b} p_{l j}^{c}\right\rangle}{2 \epsilon_{i l}}+\frac{p_{l j}^{a}\left\langle p_{j i}^{b} p_{i l}^{c}\right\rangle}{2 \epsilon_{j l}}\right\} \delta\left(\epsilon_{j i}-\omega\right) .
\end{array}
$$

In the present calculations, the function $\delta$ in Eqs. (1), (4), (5) and (9) is approximated by a Gaussian function with $\Gamma=0.2 \mathrm{eV}$. The $\mathbf{k}$ point weight $w_{\mathbf{k}}$ is set to $\left(1 / N_{k}\right)$ where $N_{k}$ is the total number of the sampled $k$-points. To obtain accurate optical properties, we perform calculations for selenium and tellurium with several different $k$-point meshes until the calculated optical properties converge to a few percent. Therefore, adequately dense $k$-point meshes of $52 \times 60 \times 1$ and $45 \times 60 \times 1$ are adopted for selenium and tellurium, respectively. Furthermore, 30 energy bands per atom are included in the present optical calculations in order to ensure that $\varepsilon^{\prime}$ and $\chi^{\prime(2)}$ obtained by the Kramer-Kronig transformation are reliable. In the slab-supercell approach, the unit cell volume $\Omega$ in Eqs. (1), (4), (5) and (9) is not well-defined for $2 \mathrm{D}$ Se and Te. In the literature, two ways have been adopted to resolve this problem. 11 18, 25] One way is to present the $2 \mathrm{D}$ response functions in terms of per unit width simply by multiplying the calculated quantities with the supercell lattice constant along the perpendicular direction [12, 16]. Physically, this means the optical responses coming from a single atomically thin film 12, 16. The other way is to use the effective unit cell volume [11, 13 15, 17, 18, 25], which is given by the area of the in-plane unit cell times the effective thickness $h$ (see Table II). This quasi bulk approach would allow one to compare the calculated quantities with that of bulk materials, and thus is adopted in this paper. Nevertheless, one should keep in mind that to evaluate the total responses from a single film, one should multiply the quantities by the effective thickness $h$ rather than light penetration depth.

\section{ELECTRONIC BAND STRUCTURE}

To understand the electronic and optical properties of 2D Se and Te materials, we plot their electronic band 

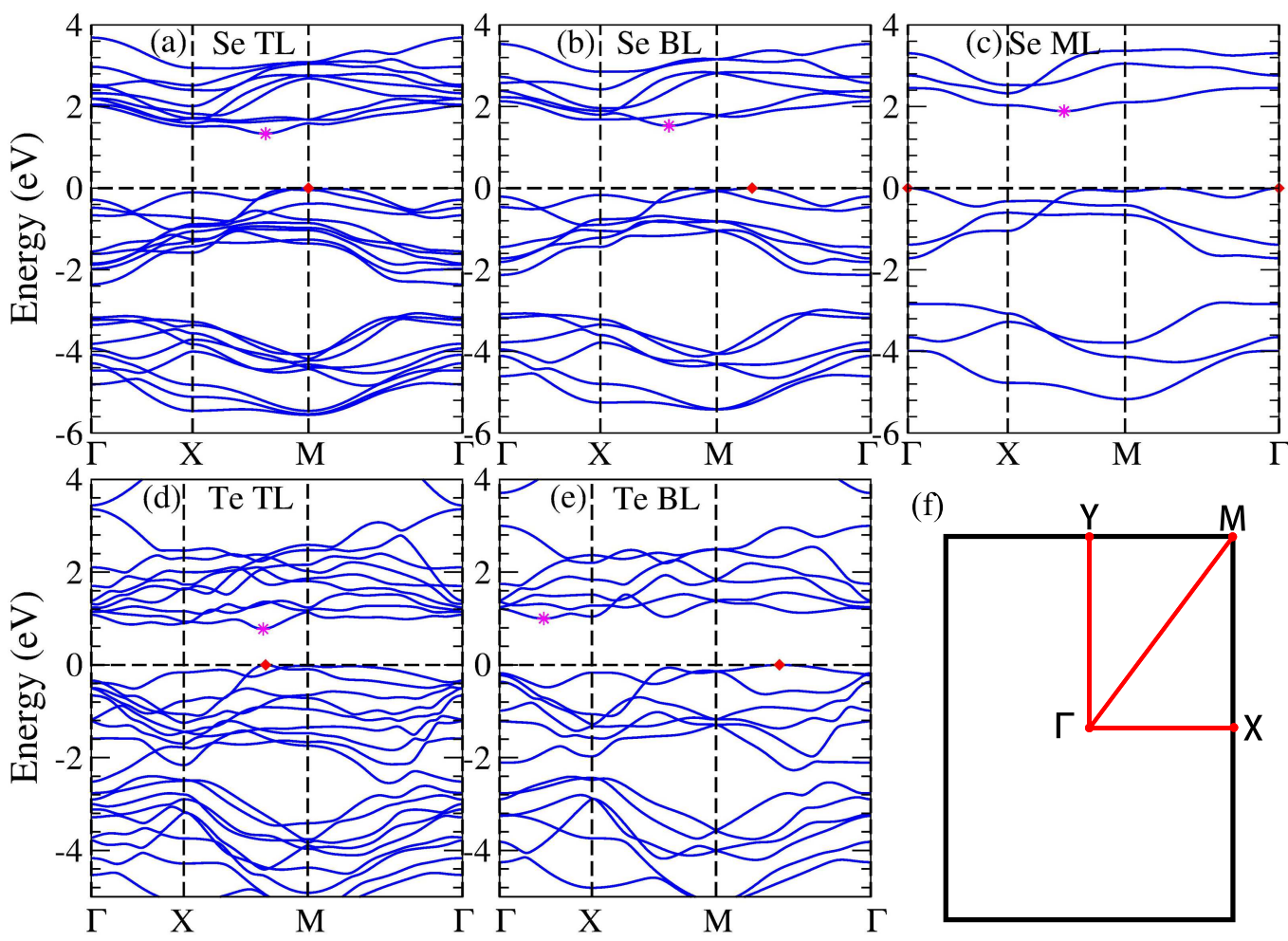

FIG. 2. Band structures of $2 \mathrm{D}$ ( $\mathrm{a}, \mathrm{b}, \mathrm{c})$ selenium as well as (d, e) tellurium. The valence band maximum (VBM) is marked as the red diamond, while the conduction band minimum is indicated by the magenta star. (f) $2 \mathrm{D}$ Brillouin zone. The VBM is at $0 \mathrm{eV}$.

TABLE III. Calculated $\left(E_{g}^{G G A}, E_{g}^{H S E-S O C}\right)$ and experimental $\left(E_{g}^{E x p}\right)$ band gaps as well as scissors operator $\left(\Delta E_{g}=\right.$ $\left.E_{g}^{H S E-S O C}-E_{g}^{G G A}\right)$ for bulk and 2D selenium and tellurium.

\begin{tabular}{|c|c|c|c|c|}
\hline & $E_{g}^{G G A}(\mathrm{eV})$ & $E_{g}^{H S E-S O C}(\mathrm{eV})$ & $E_{g}^{E x p}(\mathrm{eV})$ & $\Delta E_{g}(\mathrm{eV})$ \\
\hline Se ML & 1.857 & 2.600 & & 0.756 \\
\hline $\mathrm{BL}$ & 1.503 & 2.270 & & 0.744 \\
\hline TL & 1.305 & 2.078 & & 0.743 \\
\hline bulk & $1.002^{\mathrm{a}}$ & $1.735^{\mathrm{a}}$ & $2.0^{\mathrm{b}}$ & \\
\hline $\mathrm{Te}$ BL & 0.864 & $1.128\left(1.17^{\mathrm{c}}\right)$ & $0.85^{\mathrm{d}}$ & 0.289 \\
\hline TL & 0.654 & $1.072\left(0.95^{\mathrm{c}}\right)$ & $0.74^{\mathrm{d}}$ & 0.299 \\
\hline bulk & $0.113^{\mathrm{a}}$ & $0.322^{\mathrm{a}}$ & $0.323^{\mathrm{e}}$ & \\
\hline
\end{tabular}

Theoretical value from reference 8 .

5 Experimental value from reference 76.

cTheoretical value from reference 57.

d Experimental value from reference 56 .

5 Experimental value from reference 77.

structures in Fig. 2. Clearly, Fig. 2 shows that all the 2D Se and BL Te are indirect band gap semiconductors, while TL Te has a nearly direct band gap. For BL tellurium, the valence band maximum (VBM) is close to the $\mathrm{M}$ point along the $\mathrm{M}-\Gamma$ direction, while the conduction band minimum $(\mathrm{CBM})$ is located somewhere close to the $\Gamma$ along the $\Gamma$-X symmetry line. If the thickness of ultrathin Te film increases to that of TL, both VBM and CBM are located at a general $k$-point close to the $\mathrm{M}$ along the $\mathrm{X}-\mathrm{M}$ direction, i.e., TL Te is a nearly direct band gap semiconductor and thus may have promising applications in semiconductor devices. In contrast, a band gap transition from the direct to indirect one occurs as the thickness for the $2 \mathrm{D} \mathrm{MoS}_{2}$ film increases from ML to bulk. 75] Interestingly, when the thickness of 2D selenium increases from ML to TL, the location of the VBM moves from symmetry point $\Gamma$ to $\mathrm{M}$ along the $\Gamma-\mathrm{M}$ line. The CBM is located at a general $k$-point along the $\mathrm{X}-\mathrm{M}$ symmetry line. Nevertheless, it gets closer to the M point with the thickness [see Figs. 2(a), (b) and (c)]. Clearly, the band gap of 2D selenium is larger than that of the corresponding 2D tellurium (see Table III) due to the increased ionization energy. In general, the band gaps of $2 \mathrm{D}$ Se and Te materials are tunable by varying the number of layers.

It is well known that the band gap of a semiconductor is generally underestimated by the GGA calculations (see, e.g., Table III). Therefore, to obtain more accurate band gaps, we also calculate the band structures by using the hybrid Heyd-Scuseria-Ernzerhof (HSE) functional [78], which is known to produce much improved band gaps for semiconductors. The theoretical band gaps from the HSE and GGA calculations together with the experimental values are listed in Table III. The band 
structures from the HSE calculations with the spin-orbit coupling (SOC) included are displayed in Fig. S1. Indeed, Table III shows that the band gaps of bulk selenium and tellurium from the HSE-SOC calculations 8] agree rather well with the corresponding experimental values. Therefore, we use the band gaps from the HSESOC calculations and the scissors correction scheme 79 to evaluate linear and NLO properties for 2D Se and Te considered here. We notice that the band gap from the HSE-SOC calculation is $1.128(1.072) \mathrm{eV}$ in BL (TL) tellurium, which agrees rather well with that reported in Ref. [57] (see Table III). The small difference stems from the fact that the band gaps from Ref. [57] are based on the optB88-vdW structure, while we use the structures from the SCAN structural optimizations. It is worthwhile to point out that the gap of BL Te from the HSESOC calculations is close to $1.3 \mathrm{eV}$, the ideal gap value for perfect solar energy absorption efficiency [80]. Therefore, one could expect BL Te to have high solar energy absorption efficency, thereby leading to high power conversion efficiency for photovoltaic devices.

We notice that the experimental band gaps for BL and TL tellurium are smaller than that from our HSE-SOC calculations. This discrepancy is likely due to the effect of the graphene substrate because the environment can significantly affect the electronic properties of $2 \mathrm{D}$ materials $[81$ 83]. The graphene substrate can reduce the band gap of BL and TL tellurium mainly in two ways, namely, via (1) changing the structural parameters due to lattice mismatch and (2) offering extra dielectric screening. In particular, the in-plane lattice constants for atomically thin Te films on graphene substrate were reported [56] to be $a=5.93 \AA$ and $b=4.42 \mathrm{~A}$, which are $2.3 \%$ and $3.3 \%$ larger than that of BL Te (see Table II), respectively. It was reported [83] that the band gap of $\mathrm{MoS}_{2}$ ML would get reduced by as much as $0.46 \mathrm{eV}$ upon a lattice expansion of $2 \%$. Our GGA calculation for BL Te using the reported lattice constants [56] results in a band gap reduction of $\sim 0.1 \mathrm{eV}$. The substrates have also been found to introduce significant extra screening, thus reducing the band gap (see, e.g., Refs. 81, 82]). For example, putting a BN sheet on graphene would reduce the band gap of BN ML by $1.02 \mathrm{eV}(14 \%)$ [81]. Nonetheless, further investigations into the reduction of the band gaps of $2 \mathrm{D}$ tellurium due to substrates are beyond the scope of this paper.

\section{LINEAR OPTICAL PROPERTY}

In Fig. 3 and Fig. 4, the calculated real and imaginary parts of the optical dielectric function $\varepsilon(\omega)$ of 2D selenium and tellurium are shown, respectively. Bulk Se and Te have a trigonal lattice with the helical chains along the $c$-axis [see Figs. 1(a), 1(b) and 1(c)] and thus exhibit a rather strong uniaxial optical anisotropy [8] because of the weak inter-chain interaction. In contrast, 2D Se and Te form a $2 \mathrm{D}$ rectangular lattice with the helical chains
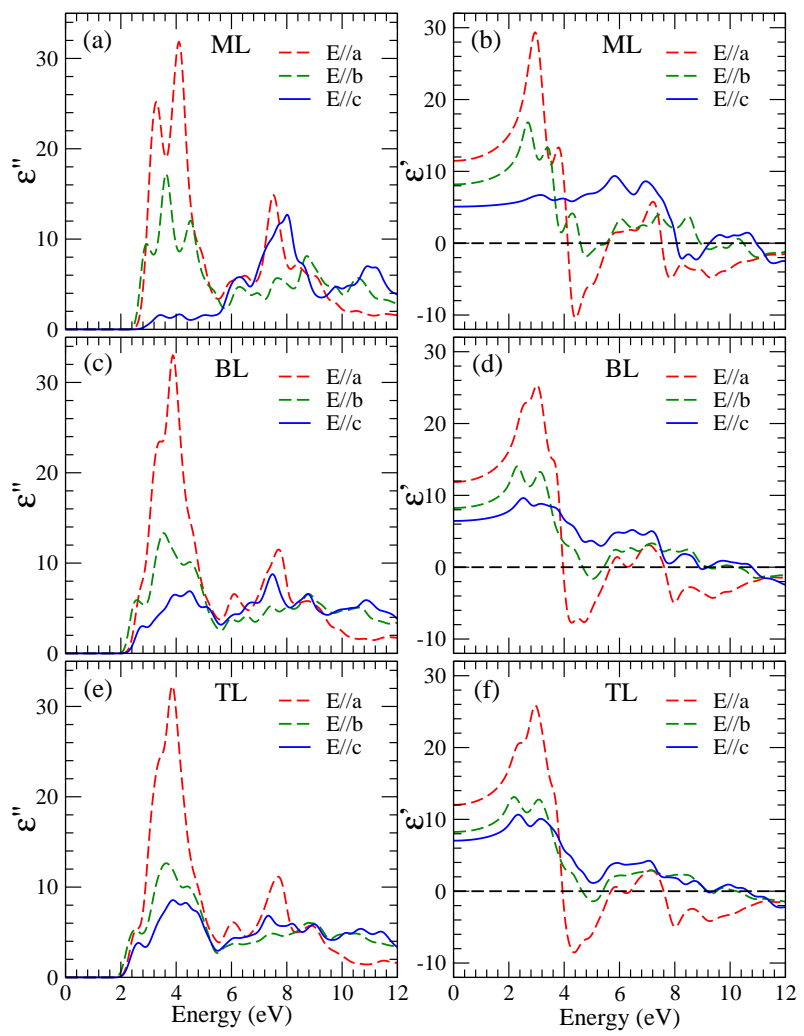

FIG. 3. The imaginary $\left[\varepsilon^{\prime \prime}(\omega)\right]$ and real part $\left[\varepsilon^{\prime}(\omega)\right]$ of the dielectric function of $(\mathrm{a}, \mathrm{b}) \mathrm{ML},(\mathrm{c}, \mathrm{d}) \mathrm{BL}$ and (e, f) TL selenium for both light polarization perpendicular (E\|a and $\mathrm{E} \| \mathrm{b})$ and parallel $(\mathrm{E} \| \mathrm{c})$ to the $c$ axis.

lying along the $a$-axis (see Fig. 1), and this may result in a strong in-plane optical anisotropy. Consequently, the real and imaginary parts of the dielectric function of 2D Se and Te compose of three independent components, namely, light polarization parallel $(E \| c)$ and perpendicular ( $E \| a$ and $E \| b$ ) to the $c$ axis.

Figure 3 shows that overall, the dielectric functions of ML, BL and TL selenium look rather similar due to the weak interlayer interaction. For example, there is a very prominant peak near $4.0 \mathrm{eV}$ in the $\varepsilon "(\omega)$ spectrum of $E \| a$ for all three multilayers. Also, in the lowenergy region of about 2.5-5.5 eV, the optical anisotropy is strong in all three multilayers. In the high-energy region of about $5.5-10 \mathrm{eV}$, there is a prominent peak and two shoulders for $E \| a$ for all three structures. For $E \| b$ and $E \| c$, the amplitude of the peaks gradually decreases with the film thickness. There are also some differences. For example, Fig. 3(a) shows that in ML Se, there is a rather sharp peak near $3.3 \mathrm{eV}$ for $E \| a$ and also near $8.0 \mathrm{eV}$ for $E \| c$ which, however, appears as a weak shoulder and bulge, respectively, in the $\varepsilon "(\omega)$ spectra of BL Se [Fig. 3(c)] and TL Se [Fig. 3(e)]. This difference may be caused by a stronger quantum confinement in ML selenium. Furthermore, Fig. 3(a) indicates that optical absorption for $E \| c$ in $2.5-5.5 \mathrm{eV}$ is very weak. 
Nevertheless, the absorption increases significantly with the film thickness [Fig. 3(c) and Fig. 3(e)]. Additionally, these peaks become oscillatory bulges in the structures, particularly in TL selenium. This could be explained by the fact that the interlayer interaction is weak but not negligible. In particular, the real and imaginary parts of the optical dielectric function $\varepsilon(\omega)$ for BL and TL selenium are rather similar except the $E \| c$ spectrum. The same phenomenon has been observed in $\mathrm{Cr}_{2} \mathrm{Ge}_{2} \mathrm{Te}_{6}$ 84]. It is noted that the optical spectra for $E \| a$ (parallel to the chain direction) and for $E \| c$ (perpendicular to the layers) of TL selenium are nearly identical to the corresponding spectra of bulk selenium [8]. In other words, the optical properties of TL selenium are like that of quasi-1D systems.
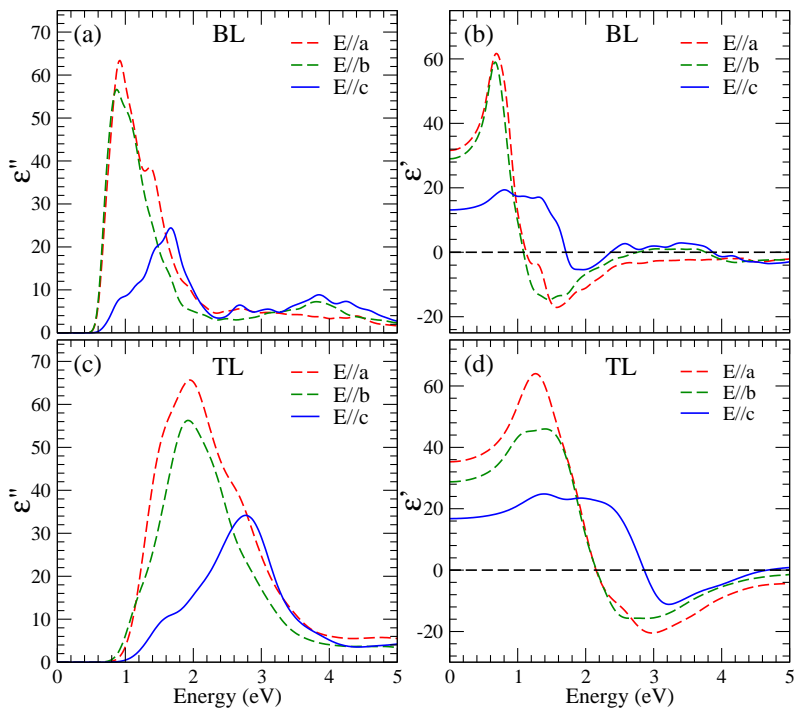

FIG. 4. The imaginary $\left[\varepsilon^{\prime \prime}(\omega)\right]$ and real part $\left[\varepsilon^{\prime}(\omega)\right]$ of the dielectric function of $(\mathrm{a}, \mathrm{b}) \mathrm{BL}$ and $(\mathrm{c}, \mathrm{d})$ TL tellurium for both light polarization perpendicular (E\|a and $\mathrm{E} \| \mathrm{b})$ and parallel $(\mathrm{E} \| \mathrm{c})$ to the $c$ axis.

As for 2D selenium, Fig. 4 indicates that overall, the dielectric functions of BL and TL tellurium are quite similar due to the weak interlayer interaction. In particular, there is a very prominant peak near $2.0 \mathrm{eV}$ in the $\varepsilon^{\prime \prime}(\omega)$ spectra of $E \| a$ and $E \| b$ for both multilayers. Furthermore, the optical spectra of $E \| a$ and $E \| b$ for both multilayers are very similar, indicating almost no in-plane optical anisotropy. This is in contrast to 2D selenium in which the peak height for $E \| b$ is much smaller than that of $E \| a$, indicating a strong in-plane optical anisotropy there. This could be explained by the fact that the relative in-plane inter-chain distance $(R / r)$ in $2 \mathrm{D}$ selenium is much larger than in 2D tellurium (see Table II). On the other hand, Te $5 p$ orbitals are much larger than Se $4 p$ orbitals, as indicated by the intrachain atomic distance ( $r$ ) (see Table II). Consequently, these result in a much stronger in-plane interchain bonding in 2D tellurium and hence a much weaker in-plane optical anisotropy. For
$E \| c$, this peak shifts to $\sim 3.4 \mathrm{eV}$ and $\sim 2.8 \mathrm{eV}$ for BL and TL tellurium [see Figs. 4(a) and 4(c)], respectively. Also, in the low-energy region up to $\sim 3.0 \mathrm{eV}$, the optical spectra for in-plane and perpendicur polarizations differ significantly in both multilayers. Above $3.0 \mathrm{eV}$, the optical spectra for all three polarizations look rather similar.

\section{SECOND HARMONIC GENERATION AND LINEAR ELECTRO-OPTIC EFFECT}

Bulk selenium and tellurium have five nonzero SHG susceptibility elements since their symmetry is either $D_{3}^{4}$ (right-handed screw) or $D_{3}^{6}$ (left-handed screw). 8] However, 2D selenium and tellurium possess a lower symmetry and thus have more nonzero SHG susceptibility elements. As mentioned above, their space group depends on the number of layers $(n)$, namely, $P 2\left(C_{2}^{1}\right)$ for the odd $n$ and $P 2_{1}\left(C_{2}^{2}\right)$ for the even $n$. Nonetheless, the two space groups have the same nonzero elements. Therefore, all 2D selenium and tellurium materials considered here have eight nonzero SHG elements as $\chi_{x x x}^{(2)}, \chi_{y x y}^{(2)}, \chi_{x y y}^{(2)}$, $\chi_{z x y}^{(2)}, \chi_{x y z}^{(2)}, \chi_{y z x}^{(2)}, \chi_{z z x}^{(2)}$ and $\chi_{x z z}^{(2)}$.

\section{A. 2D selenium}

The real and imaginary parts of the SHG coefficients for ML selenium are displayed in Fig. 5, while that of BL and TL selenium are shown, respectively, in Fig. S2 and Fig. S3 in the Supplementary Material (SM). [85] As for the linear optical property case, the calculated SHG coefficients for all the 2D Se structures are rather similar (see Figs. 5, S2 and S3) due to the weak interlayer interaction. For example, they all show that the $\chi_{x y y}^{(2)}$ spectra have the largest magnitude among the nonzero SHG matrix elements in the entire optical frequency range, and their $\chi_{x y y}^{(2)}$ spectra are similar to the $\chi_{x x x}^{(2)}$ spectra of bulk selenium [8]. They all have a prominent peak at $1.98 \mathrm{eV}$ in the $\chi_{x y y}^{\prime \prime(2)}$ spectrum. Nonetheless, this peak height get reduced from ML Se $(\sim 1420 \mathrm{pm} / \mathrm{V})$ to $\mathrm{BL}$ Se $(\sim 1020$ $\mathrm{pm} / \mathrm{V})$ and to TL Se $(\sim 840 \mathrm{pm} / \mathrm{V})$. The absolute value of SHG element $\chi_{x y y}^{(2)}$ of ML selenium at $1.98 \mathrm{eV}$ is 1423 $\mathrm{pm} / \mathrm{V}$, which is nearly 2 times larger than that of bulk selenium, and is also 6 times larger than that of $\mathrm{GaN}$ [6, 7], a widely used NLO semiconductor. Figures 5, S2 and S3 indicate that all the nonzero SHG elements of all the 2D Se structures are rather large and purely dispersive for photon energy less than half of the band gap $\left(\frac{1}{2} E_{g}\right)$. For example, $\chi_{x y y}^{(2)}(0)$ of ML selenium (see Table IV) is nearly 20 times larger than $\chi_{z z z}^{(2)}(0)$ of GaN [6, 7]. This suggests that $2 \mathrm{D}$ selenium may have potential applications in low-loss NLO optical devices.

In what follows, we take ML selenium as an example to perform a detailed analysis. In order to further analyze the prominent features in the calculated $\chi^{(2)}(\omega)$ spectra 
TABLE IV. Selenium: Static dielectric constants $\left(\varepsilon_{x}, \varepsilon_{y}, \varepsilon_{z}\right)$, nonzero SHG susceptibility elements $\left(\chi_{x x x}^{(2)}, \chi_{x y y}^{(2)}, \chi_{x z z}^{(2)}, \chi_{x y z}^{(2)}\right.$, $\left.\chi_{y z x}^{(2)}, \chi_{y x y}^{(2)}, \chi_{z z x}^{(2)}, \chi_{z x y}^{(2)}\right)(\mathrm{pm} / \mathrm{V})$, and also nonzero LEO coefficients $\left(r_{x x x}, r_{x y y}, r_{x z z}, r_{x y z}, r_{y z x}, r_{y x y}, r_{z z x}, r_{z x y}\right)(\mathrm{pm} / \mathrm{V})$.

\begin{tabular}{|c|c|c|c|c|c|c|c|c|c|c|c|c|c|c|c|c|c|c|c|}
\hline & $\varepsilon_{x}$ & $\varepsilon_{y}$ & $\varepsilon_{z}$ & $\chi_{x x x}^{(2)}$ & $\overline{\chi_{x y y}^{(2)}}$ & $\overline{\chi_{x z z}^{(2)}}$ & $\overline{\chi_{x y z}^{(2)}}$ & $\overline{\chi_{y z x}^{(2)}}$ & $\chi_{y x y}^{(2)}$ & $\chi_{z z x}^{(2)}$ & $\chi_{z x y}^{(2)}$ & $r_{x x x}$ & $r_{x y y}$ & $r_{x z z}$ & $r_{x y z}$ & $r_{y z x}$ & $r_{y x y}$ & $r_{z z x}$ & $r_{z x y}$ \\
\hline bulk & $9.0^{\mathrm{a}}$ & & $12.7^{\mathrm{a}}$ & $145^{\mathrm{a}}$ & & & 5 & & & & & $-3.58^{\mathrm{a}}$ & & & $-0.12 \mathrm{a}$ & & & & \\
\hline $\mathrm{ML}$ & 11.5 & 8.2 & 5.1 & 41 & 155 & 3 & 36 & 40 & 28 & 4 & 32 & -0.63 & -3.29 & -0.10 & -0.77 & -1.92 & -0.60 & -0.29 & -1.08 \\
\hline BL & 11.9 & 8.2 & 6.4 & 17 & 92 & -6 & 27 & 27 & 12 & -11 & 25 & -0.24 & -1.87 & 0.15 & -0.54 & -1.01 & -0.24 & 0.52 & -0.65 \\
\hline TL & 12.0 & 8.3 & 7.0 & 12 & 75 & -11 & 18 & 19 & 1 & -16 & 16 & -0.17 & -1.51 & 0.25 & -0.37 & -0.65 & -0.03 & 0.64 & -0.37 \\
\hline
\end{tabular}

$\sqrt[a]{\text { Calculated value from reference } 8 \text {. }}$

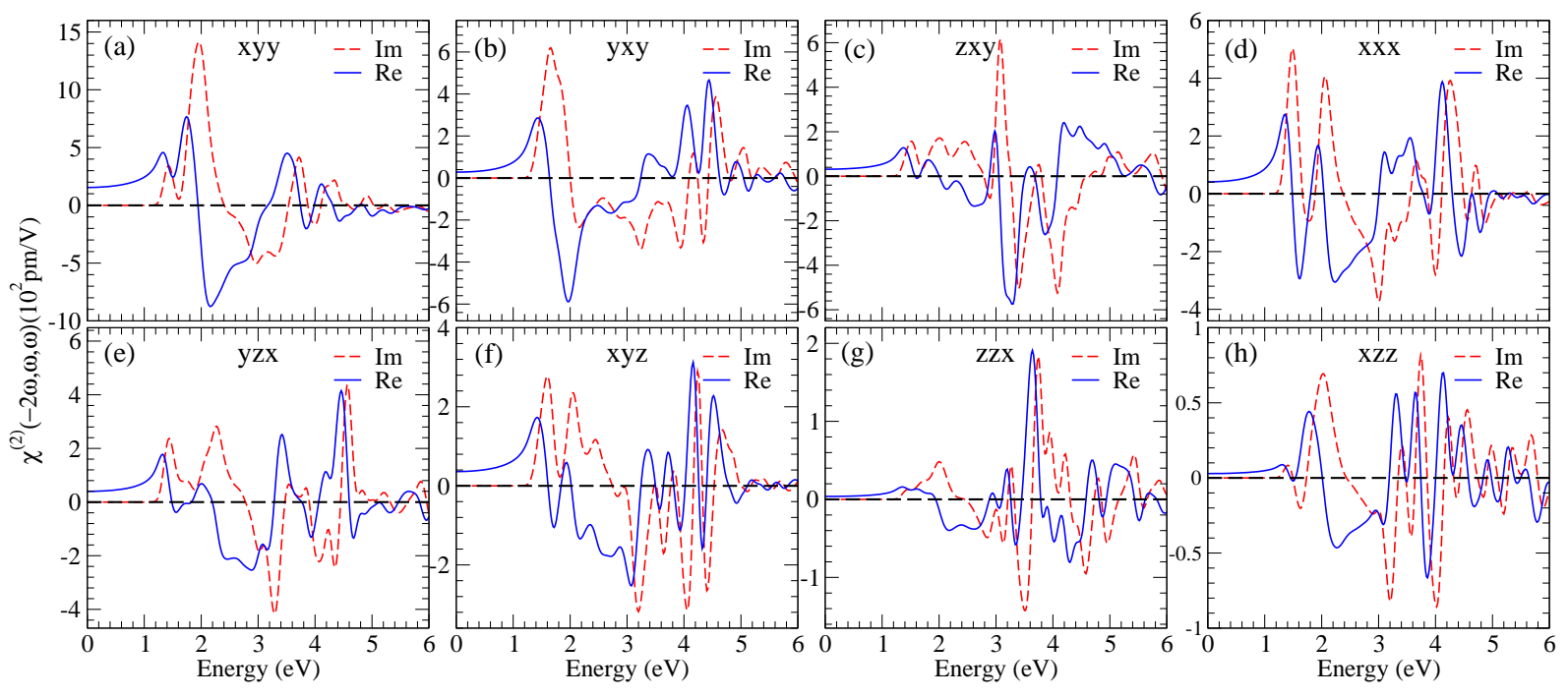

FIG. 5. Real and imaginary parts of the eight nonzero SHG susceptibility elements of ML selenium.

of ML selenium, we plot the absolute values of the imaginary part of $\chi^{(2)}$ and compare them with the absorptive part of the dielectric function $\varepsilon(\omega)$ in Fig. 6. Because they have similar features, only four elements out of the eight nonzero SHG elements are presented in Fig. 6. The SHG involves not only single-photon $(\omega)$ resonance but also double-photon $(2 \omega)$ resonance. Figure 6(a) shows that the $\chi_{x y y}^{(2)}$ spectrum can be divided into two parts. The first part from 1.22 to $2.40 \mathrm{eV}$ is primarily derived from double-photon resonances. On the other hand, the second part (above $2.40 \mathrm{eV}$ ) originates predominantly from single-photon resonances with some contribution from double-photon resonances [see Figs. 6(a) and (b)]. In this energy region, the spectra oscillate rapidly and also decrease gradually with photon energy due to the mixing of one- and two-photon resonances. Notably, for the $\left|\chi_{y x y}^{\prime \prime(2)}\right|$ spectrum, the first prominent peak at $\sim 1.70$ $\mathrm{eV}$ corresponds to the pronounced peak in the $\varepsilon_{b b}^{\prime \prime}(2 \omega)$ [see Figs. 6(c) and 6(d)], suggesting that it stems from two-photon resonances. It is clear from Fig. 6(d) that the peaks above the absorption edge of $\varepsilon_{b b}^{\prime \prime}(\omega)$ are related to the $\varepsilon_{b b}^{\prime \prime}(2 \omega)$ and $\varepsilon_{b b}^{\prime \prime}(\omega)$ spectra, indicating that they can be caused by both double- and single-photon resonances. Figures $6(\mathrm{e})$ and $6(\mathrm{~g})$ show that the peaks in $\chi_{z z x}^{(2)}$ and $\chi_{x z z}^{(2)}$ from the absorption edge of $\varepsilon_{c c}^{\prime \prime}(2 \omega)$ to the absorption edge of $\varepsilon_{c c}^{\prime \prime}(\omega)$ may be attributed to the two-photon resonances with $\mathrm{E} \| \mathrm{c}$ [cf. $\left.\varepsilon_{c c}^{\prime \prime}(2 \omega)\right]$. Above the absorption edge of $\varepsilon_{c c}^{\prime \prime}(\omega)$, the absolute values of the imaginary part of $\chi^{(2)}(\omega)$ oscillate rapidly. This is consistent with the fact that in this energy region $\chi^{(2)}(\omega)$ comes mainly from two-photon resonances with some contribution from onephoton resonances. Clearly, the modulus of the imaginary part of $\chi_{x y y}^{\prime \prime(2)}$ is larger than that of $\chi_{y x y}^{\prime \prime(2)}$, and is also much larger than that of $\chi_{z z x}^{\prime \prime(2)}$ and $\chi_{x z z}^{\prime \prime(2)}$. Interestingly, this can be correlated with the magnitude relationship of $\varepsilon_{a a}^{\prime \prime}(\omega), \varepsilon_{b b}^{\prime \prime}(\omega)$ and $\varepsilon_{c c}^{\prime \prime}(\omega)\left[\right.$ or $\varepsilon_{a a}^{\prime \prime}(2 \omega), \varepsilon_{b b}^{\prime \prime}(2 \omega)$ and $\left.\varepsilon_{c c}^{\prime \prime}(2 \omega)\right]$.

The calculated static SHG susceptibility $\chi^{(2)}(0)$ and LEO coefficient $r(0)$ as well as dielectric constant $\varepsilon(0)$ of bulk and 2D selenium are presented in Table IV. As for the optical frequency case discussed above, the static SHG susceptibility of 2D selenium is also highly anisotropic. In particular, $\chi_{x y y}^{(2)}(0)$ of ML selenium is over 50 times larger than $\chi_{x z z}^{(2)}(0)$ (see Table IV). Nevertheless, Table IV indicates that the anisotropy in the LEO coefficient $r(0)$ gets much reduced because of the strong anisotropy of the static $\varepsilon(0)$ [See Eq. (7)]. Remarkably, LEO coefficient $r_{x y y}(\sim 3.29 \mathrm{pm} / \mathrm{V})$ of ML Se 

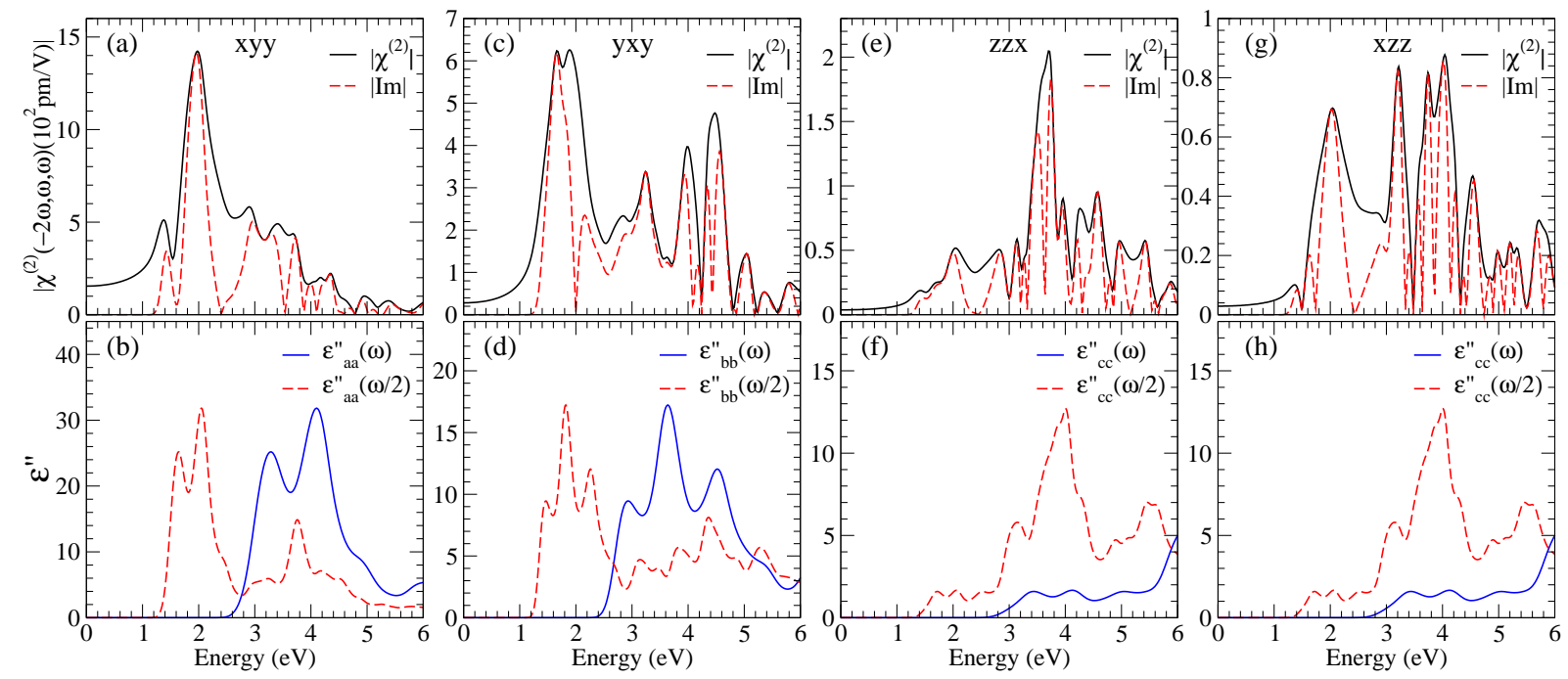

FIG. 6. Absolute value of nonzero SHG susceptibility elements (a) $\chi_{x y y}^{(2)}$, (c) $\chi_{y x y}^{(2)}$, (e) $\chi_{z z x}^{(2)}$ and (g) $\chi_{x z z}^{(2)}$ of ML selenium. Imaginary part $\varepsilon^{\prime \prime}(\omega)$ of the dielectric function for light polarization (b, d) perpendicular and (f, h) parallel to the $c$ axis.

is nearly 6 times larger than that of bulk GaN polytypes [6, 7], suggesting that $2 \mathrm{D}$ selenium may become promising electric-optic materials for opto-electronic devices.

\section{B. 2D tellurium}

The calculated static dielectric constant $\varepsilon(0)$, SHG susceptibility $\chi^{(2)}(0)$ and LEO coefficient $r(0)$ of $2 \mathrm{D}$ and bulk tellurium are listed in Table V. First of all, we notice that 2D Te exhibit much stronger static SHG responses than 2D Se. In particular, $\chi_{x y y}^{(2)}(0)$ for BL Te (Table V) is more than 100 times larger than that of GaN in both zinc-blende and wurtzite structures [6, 7]. Thus, our calculations indicate that $2 \mathrm{D}$ Te may have valuable NLO applications such as frequency doublers. Nevertheless, as for $2 \mathrm{D}$ selenium, LEO coefficient $\left(r_{y z x}\right)$ of BL Te is only 5 times greater than that of GaN [6, 7], because static dielectric constant $\varepsilon(0)$ of $\mathrm{BL}$ Te also becomes larger than BL Se [see Tables IV and V as well as Eq. (7)].

The calculated real and imaginary parts of the SHG susceptibility of TL and BL tellurium are displayed, respectively, in Fig. 7 and Fig. S4 in the SM [85]. Like $2 \mathrm{D}$ selenium, the spectral shape of the real and imaginary parts of $\chi^{(2)}(\omega)$ for BL and TL tellurium are rather similar, indicating a weak interlayer interaction. Nevertheless, unlike 2D selenium, the $\chi_{x x x}^{(2)}(\omega)$ spectrum has the largest magnitude in 2D tellurium (see Fig. 7). Furthermore, when one moves from BL tellurium (Fig. S4) to TL tellurium (Fig. 7), the magnitude of the $\chi_{x x x}^{(2)}(\omega)$ spectrum increases significantly rather than decreases. Another striking difference is that the $\chi^{(2)}(\omega)$ spectra of $2 \mathrm{D}$ tellurium in general are more than 10 times larger than the corresponding spectra in 2D selenium (see, e.g., Figs. 7 and S3). In particular, the peak height near 1.2
$\mathrm{eV}$ in the $\chi_{x x x}^{\prime \prime(2)}(\omega)$ spectrum of TL selenium [Fig. S3(g)] more than 40 times smaller than that of TL tellurium [Fig. 7(a)]. For a specific semiconductor, the smaller the band gap is, the larger the magnitude of the second-order NLO susceptibility, as can be seen from Eqs. (4) and (5). This is the main reason why $2 \mathrm{D}$ tellurium generally have much larger SHG coefficients than 2D selenium (see also Tables IV and V) because of the much smaller band gaps of $2 \mathrm{D}$ tellurium.

Let us now focus on the $\chi_{x x x}^{(2)}$ spectrum since it is the largest nonzero element among all the nonvanishing SHG coefficients of $2 \mathrm{D}$ tellurium. Figure 7 (a) shows that $\chi_{x x x}^{(2)}$ is large in the photon energy range from $0.0 \mathrm{eV}$ to $\sim 1.86$ $\mathrm{eV}$. In the energy range from 0.0 to $0.22 \mathrm{eV}$, the real part of the $\chi_{x x x}^{(2)}$ remains almost constant. As photon energy further increases, it increases steadily, and produces a small peak at $\sim 0.58 \mathrm{eV}$ [see Fig. 7(a)]. In the energy region of $0.70-1.02 \mathrm{eV}$, the spectrum of $\chi_{x x x}^{\prime(2)}$ becomes negative and forms a huge negative peak at $\sim 0.86$ $\mathrm{eV}$. Beyond $1.02 \mathrm{eV}$, it becomes positive again and decreases gradually in the higher energy region. The imaginary part of $\chi_{x x x}^{(2)}$ forms a sine-function shape in the photon energy range from $\sim 0.5$ to $1.7 \mathrm{eV}$ with a large maximum of $12000 \mathrm{pm} / \mathrm{V}$ at $\sim 0.7 \mathrm{eV}$ and a large minimum of $-14000 \mathrm{pm} / \mathrm{V}$ at $\sim 1.0 \mathrm{eV}$ [see Fig. $7(\mathrm{a})$ ]. It is negligible outside this photon energy range. Interestingly, all the nonzero SHG coefficients of TL tellurium have nearly identical shapes (see Fig. 7). Finally, we note that the magnitude of $\left|\chi_{x x x}^{(2)}(-2 \omega, \omega, \omega)\right|$ at $0.98 \mathrm{eV}$ is as high as $15000 \mathrm{pm} / \mathrm{V}$, which is 4 times larger than that of bulk Te [8] and 65 times larger than that of GaN [6, 7]. All these suggest that few-layer tellurium would find promising applications in, e.g., optical switching, ultrathin second-harmonic and sum frequency generation 
TABLE V. Tellurium: Static dielectric constants $\left(\varepsilon_{x}, \varepsilon_{y}, \varepsilon_{z}\right)$, nonzero SHG susceptibility elements $\left(\chi_{x x x}^{(2)}, \chi_{x y y}^{(2)}, \chi_{x z z}^{(2)}, \chi_{x y z}^{(2)}\right.$, $\left.\chi_{y z x}^{(2)}, \chi_{y x y}^{(2)}, \chi_{z z x}^{(2)}, \chi_{z x y}^{(2)}\right)(\mathrm{pm} / \mathrm{V})$ and also nonzero LEO coefficients $\left(r_{x x x}, r_{x y y}, r_{x z z}, r_{x y z}, r_{y z x}, r_{y x y}, r_{z z x}, r_{z x y}\right)(\mathrm{pm} / \mathrm{V})$.

\begin{tabular}{|c|c|c|c|c|c|c|c|c|c|c|c|c|c|c|c|c|c|c|c|}
\hline & $\varepsilon_{x}$ & $\varepsilon_{y}$ & $\varepsilon_{z}$ & $\chi_{x x x}^{(2)}$ & $\chi_{x y y}^{(2)}$ & $\overline{\chi_{x z z}^{(2)}}$ & $\overline{\chi_{x y z}^{(2)}}$ & $\chi_{y z x}^{(2)}$ & $\overline{\chi_{y x y}^{(2)}}$ & $\overline{\chi_{z z x}^{(2)}}$ & $\overline{\chi_{z x y}^{(2)}}$ & $r_{x x x}$ & $r_{x y y}$ & $r_{x z z}$ & $r_{x y}$ & $r_{y z x}$ & $r_{y x y}$ & $r_{z z x}$ & $r_{z x y}$ \\
\hline$\overline{\text { bulk }}$ & $33.2^{\mathrm{a}}$ & & $49.4^{\mathrm{a}}$ & $169^{\mathrm{a}}$ & & & $1009^{\mathrm{a}}$ & & & & & -0.3 [a & & & $-1.82^{\mathrm{a}}$ & & & & \\
\hline BL & 31.6 & 29.0 & 13.2 & 557 & 1077 & 31 & 592 & 585 & 897 & 0 & 591 & -1.11 & -2.34 & -0.15 & -1.29 & -3.06 & -1.95 & 0 & -2.84 \\
\hline $\mathrm{TL}$ & 35.3 & 28.8 & 16.8 & 823 & 872 & 18 & 656 & 644 & 683 & -11 & 626 & -1.32 & -1.72 & -0.06 & -1.29 & -2.67 & -1.34 & 0.08 & -2.11 \\
\hline
\end{tabular}

[Calculated value from reference 8 .

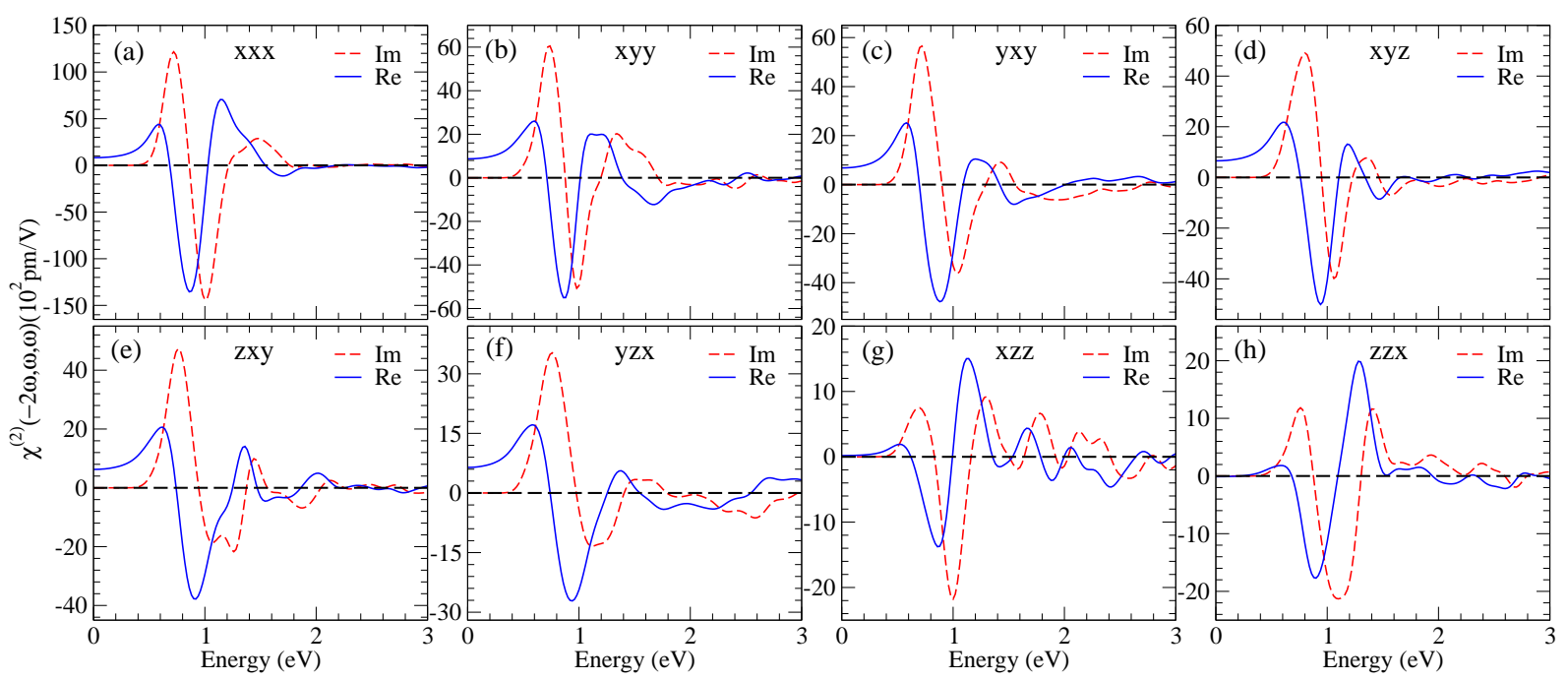

FIG. 7. Real and imaginary parts of the eight nonzero SHG susceptibility elements of TL tellurium.

devices, optical modulation, and light signal modulators.

In order to further understand the features in the calculated SHG spectra, the absolute values of the imaginary parts of four prominent elements $\chi_{x x x}^{(2)}, \chi_{x y y}^{(2)}, \chi_{x z z}^{(2)}$ and $\chi_{z z x}^{(2)}$ of TL tellurium are plotted in Fig. 8 as examples, together with the imaginary part of the dielectric function. Figure 8 suggests that the spectral feature of both $\chi_{x z z}^{(2)}$ and $\chi_{z z x}^{(2)}$ between $\sim 0.44$ and $\sim 1.72 \mathrm{eV}$ could be attributed to the two-photon resonances shown in $\varepsilon_{c c}^{\prime \prime}(2 \omega)$. In contrast, the structure above $\sim 1.72 \mathrm{eV}$ stems from both single-photon [see $\varepsilon_{c c}^{\prime \prime}(\omega)$ ] and two-photon resonances $\left[\operatorname{see} \varepsilon_{c c}^{\prime \prime}(2 \omega)\right]$. As a result, the SHG spectra, especially $\chi_{x z z}^{(2)}$, oscillate rapidly and diminish gradually in the higher energy range [see Figs. 8(e) and 8(g)]. Similarly, for $\chi_{x x x}^{(2)}$ and $\chi_{x y y}^{(2)}$, the spectral structures between $\sim 0.34 \mathrm{eV}$ and $\sim 1.36 \mathrm{eV}$ are mainly due to double-photon $(2 \omega)$ resonances for $E \| a$ and $E \| b$ [see $\varepsilon_{a a}^{\prime \prime}(2 \omega)$ and $\left.\varepsilon_{b b}^{\prime \prime}(2 \omega)\right]$, respectively. The features in $\chi_{x x x}^{(2)}$ and $\chi_{x y y}^{(2)}$ for photon energies above $1.36 \mathrm{eV}$ could be attributed to the single-photon resonances as can be seen from $\varepsilon_{a a}^{\prime \prime}(\omega)$ and $\varepsilon_{b b}^{\prime \prime}(\omega)$, respectively. Clearly, the magnitude of $\chi_{x x x}^{(2)}$ is largest, and also is about several times larger than the other nonzero elements (see Fig. 7). Interestingly, this seems to be correlated with the fact that the magnitude of $\varepsilon_{a a}^{\prime \prime}(\omega)\left[\varepsilon_{a a}^{\prime \prime}(2 \omega)\right]$ is larger than that of $\varepsilon_{b b}^{\prime \prime}(\omega)\left[\varepsilon_{b b}^{\prime \prime}(2 \omega)\right]$, and much larger than $\varepsilon_{c c}^{\prime \prime}(\omega)\left[\varepsilon_{c c}^{\prime \prime}(2 \omega)\right]$.

\section{BULK PHOTOVOLTAIC EFFECT}

The generation of photocurrents is a crucial component for solar energy harvesting. A promising mechanism for the photocurrent generation is the BPVE in which DC currents are generated in a noncentrosymmetric material under light irradiation. Young et al. recently demonstrated that the shift current dominates the BPVE in $\mathrm{BaTiO}_{3}$ 22]. In this section, we present the calculated nonzero elements of the shift current conductivity tensor for all the 2D selenium and tellurium considered here.

We notice that nonzero elements of the shift current conductivity tensor are the same as that of the SHG susceptibility tensor. Therefore, for all the 2D selenium and tellurium considered here, the nonzero elements are $\sigma_{x x x}, \sigma_{y x y}, \sigma_{x y y}, \sigma_{z x y}, \sigma_{x y z}, \sigma_{y z x}, \sigma_{z z x}$ and $\sigma_{x z z}$. However, since we consider only the shift current generation due to the linearly polarized light here, we will present only the calculated shift current elements of $\sigma_{x x x}, \sigma_{x y y}$ and $\sigma_{x z z}$ in this paper. Furthermore, here we have also calculated the shift current conductivity tensors for bulk selenium and bulk tellurium for comparison. The computational details were already reported in Ref. [8]. Their 


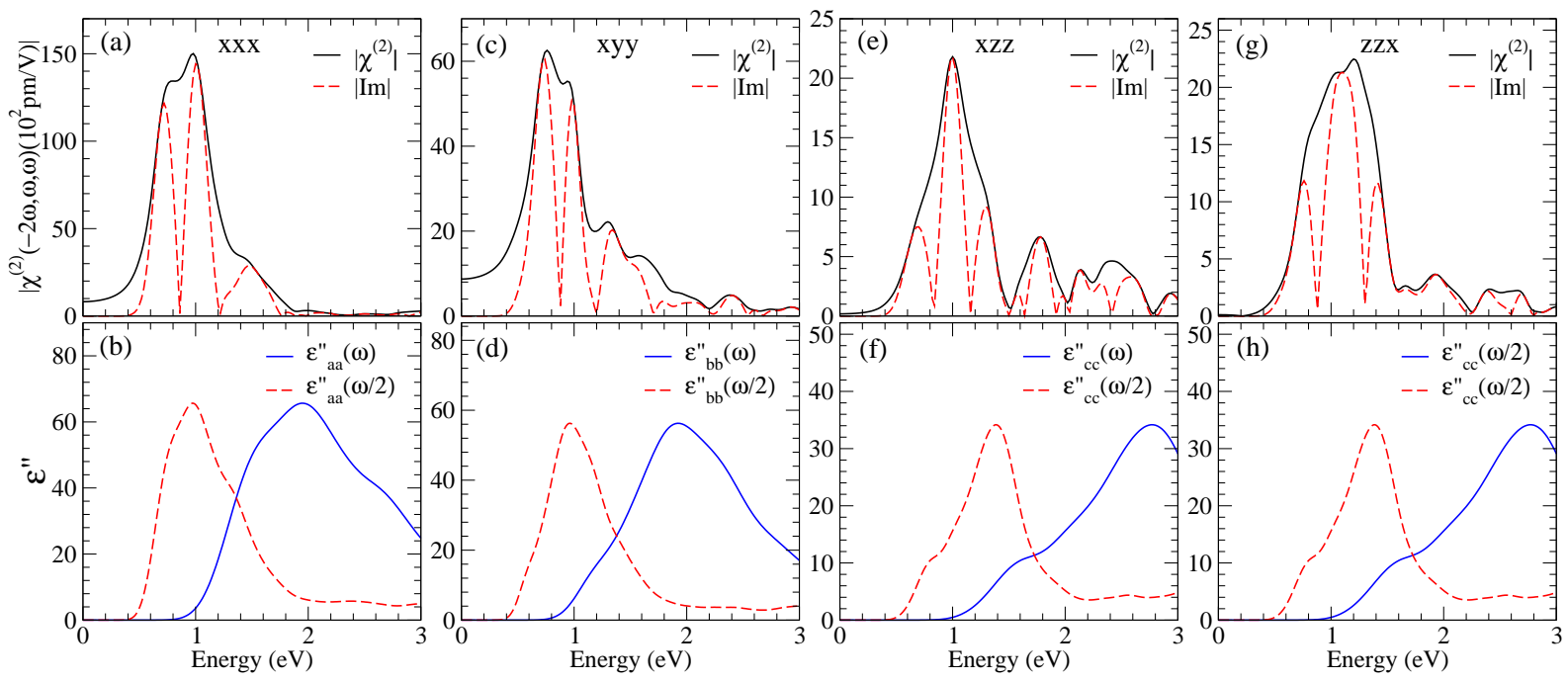

FIG. 8. Absolute values of nonzero SHG susceptibility elements (a) $\chi_{x x x}^{(2)}$, (c) $\chi_{x y y}^{(2)},(\mathrm{e}) \chi_{x z z}^{(2)}$ and (g) $\chi_{z z x}^{(2)}$ of TL tellurium. Imaginary part $\varepsilon^{\prime \prime}(\omega)$ of the dielectric function for light polarization (b, d) perpendicular and (f,h) parallel to the $c$ axis.

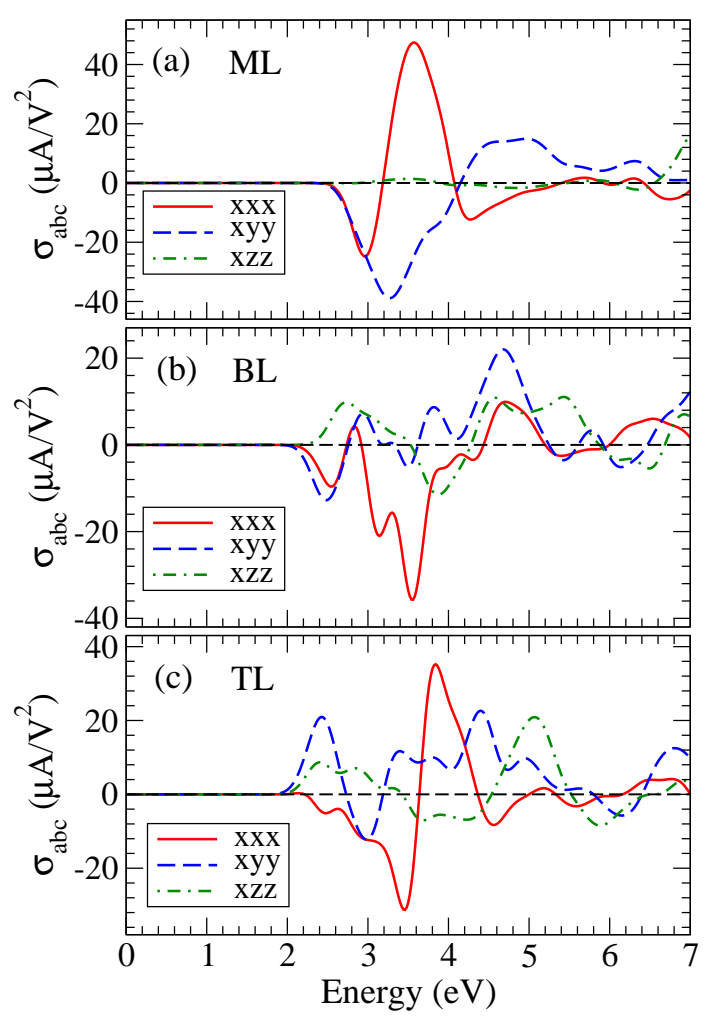

FIG. 9. Shift current conductivity $(\sigma)$ versus photon energy for (a) bulk selenium and (b) bulk tellurium.

space group is $P 3_{1} 21$ and there are two independent shift current elements among the five nonzero elements, i.e., $\sigma_{x x x}=-\sigma_{x y y}=-\sigma_{y x y}$ and $\sigma_{x y z}=-\sigma_{y z x}$. The calculated two independent shift current elements of bulk selenium and bulk tellurium are displayed in Fig. 9.

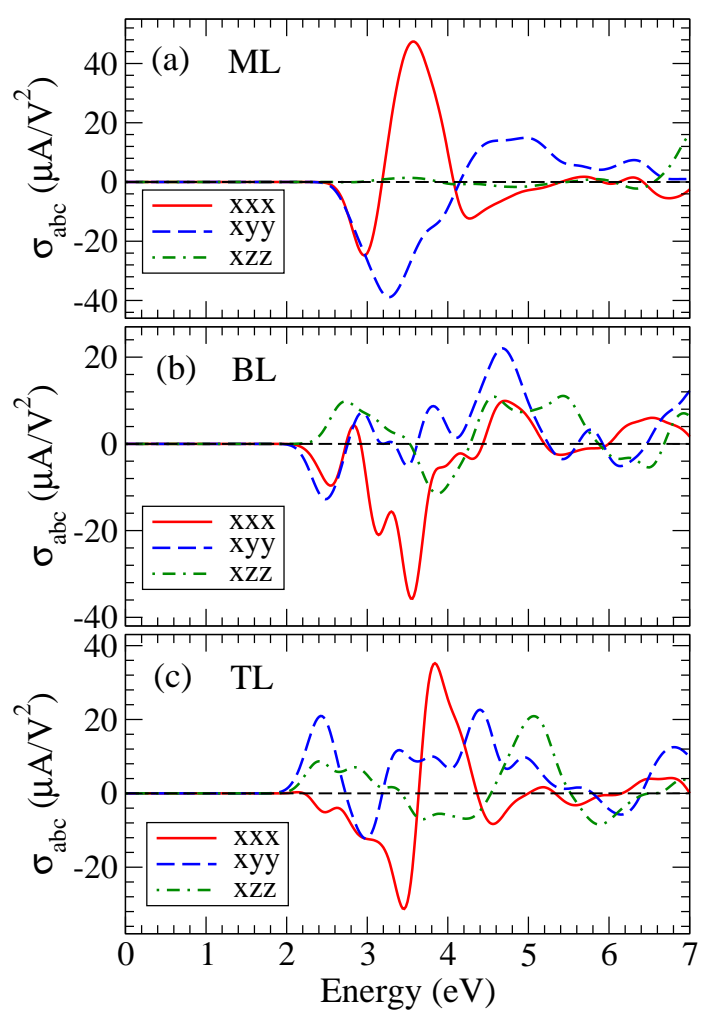

FIG. 10. Shift current conductivity $(\sigma)$ versus photon energy for (a) ML, (b) BL and (c) TL selenium.

\section{A. 2D selenium}

The calculated shift current $\sigma_{a b c}$ spectra of $2 \mathrm{D}$ selenium are plotted in Fig. 10. It is clear that unlike the dielectric function and SHG spectra, the shift current 
spectra exhibit a strong layer-thickness dependence. Furthermore, the $\sigma_{x x x}$ spectrum in general has the largest magnitude and the $\sigma_{x z z}$ has the smallest magnetiude. In particular, the $\sigma_{x z z}$ spectrum of ML selenium is negligibly small compared with the $\sigma_{x x x}$ and $\sigma_{x y y}$ spectra [see Fig. 10(a)]. This is clearly due to the very small absorption of light polarized along the layer normal direction in this photon energy range [see Fig. 3(a)]. As the layer thickness increases when one moves from ML selenium to BL and TL selenium, the optical absorption of the outof-plane polarized light increases (see Fig. 3) and hence the magnitude of the $\sigma_{x z z}$ spectrum also increases (see Fig. 10). Interestingly, the height $\left(47 \mu \mathrm{A} / \mathrm{V}^{2}\right)$ of the maximal peak of the $\sigma_{x x x}$ spectrum at $3.58 \mathrm{eV}$ of ML selenium is larger than that of the $\sigma_{x x x}$ spectrum at 3.56 $\mathrm{eV}\left(-36 \mu \mathrm{A} / \mathrm{V}^{2}\right)$ for BL selenium and also at $3.84 \mathrm{eV}(35$ $\left.\mu \mathrm{A} / \mathrm{V}^{2}\right)$ for $\mathrm{TL}$ selenium. We notice that the magnitudes of the $\sigma_{x x x}$ spectra of $2 \mathrm{D}$ selenium are at least comparable to that of the $\sigma_{z x x}$ and $\sigma_{z z z}$ spectra of $\mathrm{BaTiO}_{3}$ [22], an archetypical ferroelectric BPVE material. These magnitudes are also comparable to that of ML group-IV monochalcogenides (e.g., GeS) 25]. Therefore, 2D selenium may become excellent materials for photovoltaic solar cells. Figures $9(\mathrm{a})$ and 10 indicate that the shift current conductivities of bulk and 2D selenium are in the same order of magnitude, although their spectral shapes are quite different.

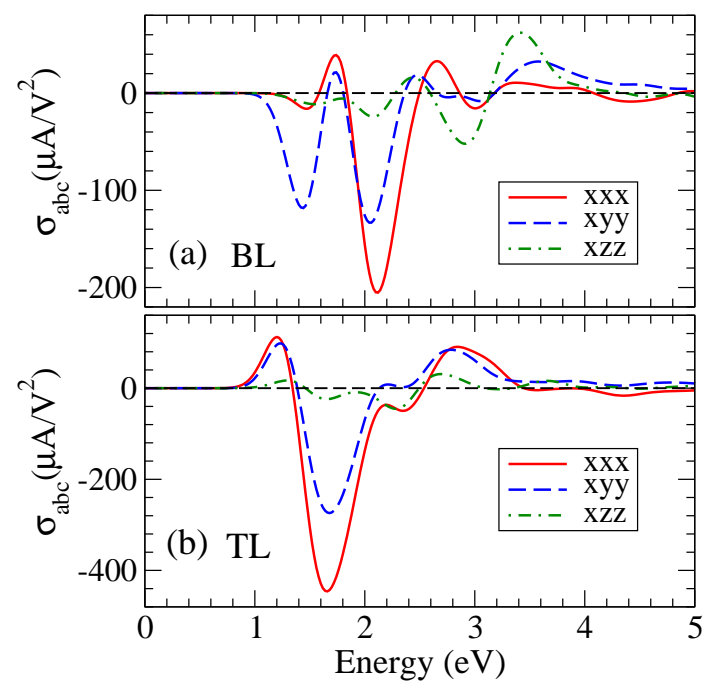

FIG. 11. Shift current conductivity $(\sigma)$ versus photon energy for (a) BL and (b) TL tellurium.

\section{B. $2 \mathrm{D}$ tellurium}

We display the calculated shift current conductivity $\left(\sigma_{a b c}\right)$ spectra of BL and TL tellurium in Fig. 11. First of all, we notice that the $\sigma_{a b c}$ spectra of 2D tellurium are large in the optical frequency range, being nearly one- order of magnitude larger than the corresponding spectra of $2 \mathrm{D}$ selenium (see Fig. 10). This notable difference between 2D tellurium and 2D selenium may be attributed to the smaller band gaps and highly covalent bonding in $2 \mathrm{D}$ tellurium. Furthermore, the magnitude of the $\sigma_{x x x}$ at $1.66 \mathrm{eV}$ of TL tellurium is nearly 4 times larger than the maximum of the shift current conductivities of ML GeS [25]. In fact, the shift current conductivity of ML Ge and $\mathrm{Sn}$ monochalcogenides apparently is larger than that of other noncentrosymmetric semiconductors reported so far [25]. This indicates that 2D tellurium are very promising materials for photovoltaic devices. The $\sigma_{x x x}$ spectrum in general has the largest magnitude while the $\sigma_{x z z}$ has the smallest magnetiude. Indeed, the $\sigma_{x z z}$ spectrum of TL tellurium is negligibly small compared with the $\sigma_{x x x}$ and $\sigma_{x y y}$ spectra [see Fig. 11(b)]. Second, unlike the dielectric function and SHG spectra, the shift current spectra exhibit a strong layer-thickness dependence. In particular, the height of the prominent negative peak at $\sim 1.7 \mathrm{eV}$ in the $\sigma_{x z z}$ spectrum of TL tellurium is nearly twice as large as that of the negative peak at $\sim 2.1 \mathrm{eV}$ in BL tellurium (see Fig. 11). In contrast to 2D selenium, 2D tellurium have the shift current conductivities that are much larger than bulk tellurium (see Figs. 9(b) and 11). In particular, the shift current conductivities of TL Te [Fig. 11(b)] are as much as five times larger than that of bulk tellurium [Fig. 9(b)]. Our work thus demonstrates that few-layer tellurium with narrow band gaps are promising materials for high efficient photovoltaic solar cells.

\section{DISCUSSION}

For a specific semiconductor, low frequency NLO properties depend significantly on its band gap. This could be seen from the fact that the energy differences between the initial and final states of the optical transitions are in the denominators of Eqs. (4), (5) and (9). This indicates that the smaller the band gap is, the larger the magnitude of the NLO responses would be. For example, for the imaginary part of the SHG coefficients, the magnitude in the low frequency region would be roughly proportional to the inverse of the fourth power of the band gap [see Eqs. (4) and (5)]. Equation (9) also indicates that the magnitude of the shift current conductivity is roughly proportional to the inverse of the third power of the band gap. As a result, although 2D Se and Te have identical crystalline structures and similar band structures, the calculated imaginary part of the dielectric function, SHG coefficients and shift photoconductivities for 2D Te are much larger than that of 2D Se simply because 2D Te has a much smaller band gap. Interestingly, unlike bulk Te, few-layer Te have a band gap being larger than the optical frequencies of interest, showing that the materials would be useful for the NLO applications.

As mentioned in the preceding sections, compared to other semiconductors with similar band gaps, 2D Se and 
(a) TL Se

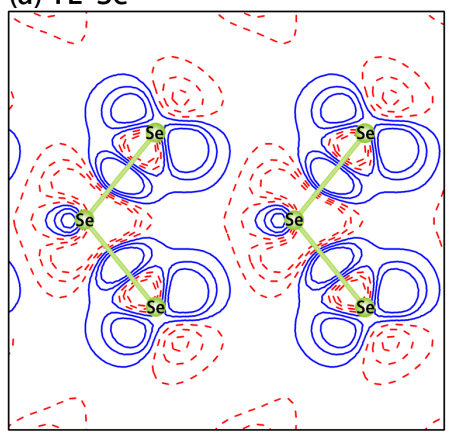

(d) TL Te

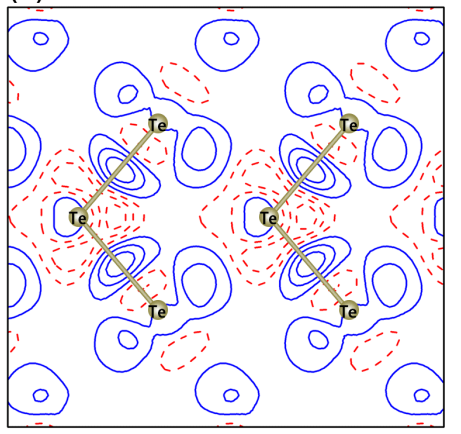

(b) $\mathrm{BL} \mathrm{Se}$

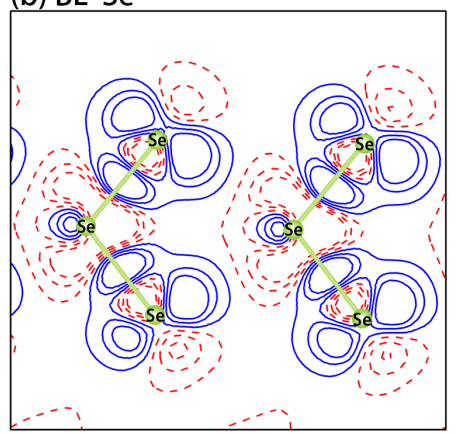

(e) $\mathrm{BL} \mathrm{Te}$ (c) ML Se

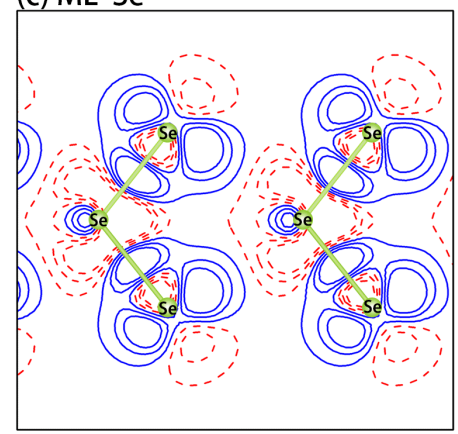

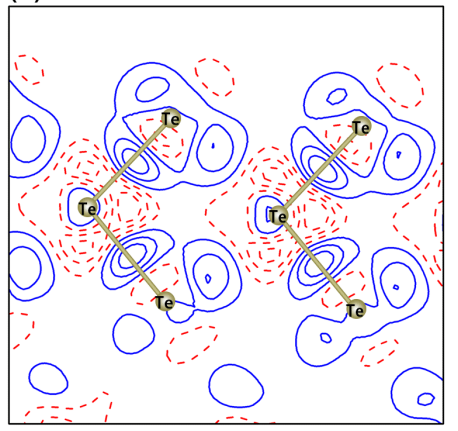

FIG. 12. Contour plots of the deformation charge density in (a) TL, (b) BL and (c) ML selenium as well as in (d) TL and (e) BL tellurium. The contour interval is $0.02 \mathrm{e} / \AA^{3}$. Positive contours (blue solid lines) indicate electron accumulation and negative contours (red dashed lines) depict electron depletion.

Te generally exhibit much larger NLO effects. To further understand the origins of the enhanced NLO responses of 2D selenium and tellurium, we calculate the difference charge density which is defined as the difference between the total charge density and the superposition of the free atomic charge densities, as displayed in Fig. 12. Figures 12(a), 12(b) and 12(c) [Figs. 12(d) and 12(e)] show that a considerable electron charge buildup occurs in the vicinity of the Se-Se (Te-Te) bond center by depleting the charge around the atoms along the bond directions in 2D selenium (2D tellurium). This indicates that there is strong directional covalent bonding in these 2D materials. It is known that strong covalent bonding would generate large spatial overlap between the wave functions of initial and final states and thus leads to large optical matrix elements, thereby resulting in enhanced NLO responses. Furthermore, Fig. 12 also shows a charge buildup around each atom in the direction perpendicular to the chain, indicating the existence of lone-pair electrons. The presence of lone-pair electrons is beneficial to the generation of induced dipole oscillations by the optical electric fields, thus leading to larger $\chi^{(2)}$ values [86, 87] and other NLO effects. We notice that these features in the difference charge density distributions in 2D Se and Te are quite similar to that found in bulk trigonal Se and Te [8]. Bulk Se and Te crystalize in helical chainlike structures and also exhibit large SHG and LEO effect [8]. Therefore, in general, quasi one-dimensional crystals with strong di- rectional covalency and lone-pair electrons would possess large NLO values. Moreover, high anisotropy would result in large joint DOS, and hence give rise to larger BPV and SHG effect 88, 89.

Therefore, in general, to search for the materials with large NLO responses, one first should focus on those semiconductors with smallest possible band gaps which are larger than the optical frequencies required by specific NLO applications. Second, one should also pay attention to low dimensional systems with high anisotropy, strong covalency and/or lone-pair electrons.

\section{CONCLUSION}

We have carried out a systematic first-principles investigation on the nonlinear optical properties of 2D selenium and tellurium within the generalized gradient approximation plus scissors correction. We find that all the $2 \mathrm{D}$ materials possess large NLO responses. In particular, TL Te exhibits a SHG coefficient $\chi_{x x x}^{(2)}$ of up to $15000 \mathrm{pm} / \mathrm{V}$, which is 65 times larger than that of bulk GaN. BL Te exhibits gigantic static SHG coefficient $\chi_{x y y}^{(2)}$, which is more than 100 times larger than that of bulk GaN. ML Se also displays prominent NLO effects with maximal nonvanishing SHG coefficient $\chi_{x y y}^{(2)}$ being as large as $\sim 1400 \mathrm{pm} / \mathrm{V}$, which exceeds 6 times 
larger than that of GaN. Furthermore, ML Se and BL Te have large LEO coefficients $r_{x y y}$ and $r_{y z x}$, being up to 6 times and 5 times larger than that of bulk GaN polytypes, respectively. We also find that the maximum of the shift current in the visible frequency range for TL Te is two times larger than that of ML GeS. All these findings suggest that 2D selenium and tellurium are promising NLO materials for applications in, e.g., photovoltaic devices, second-harmonic generation, sum frequency generation, frequency conversion, phase matching, electro-optical switches and light signal modulators. The features in the SHG spectra of the 2D Se and Te are correlated with the peaks in the imaginary part of the corresponding optical dielectric function in terms of single-photon and double-photon resonances. We believe that this work will stimulate further experiments on the second-order nonlinear optical responses of these fascinating elemental 2D materials.

\section{ACKNOWLEDGEMENTS}

M. C. thanks Department of Physics and Center for Theoretical Physics, National Taiwan University for its hospitality during her three months visit. Work at Xiamen University is supported by the National Key R\&D Program of China (Grant No. 2016YFA0202601), and the National Natural Science Foundation of China (No. 11574257). G. Y. G. acknowledges support from the Ministry of Science and Technology, the Academia Sinica, the National Center for Theoretical Sciences in Taiwan.
[1] Y. R. Shen, The Principle of Nonlinear Optics (John Wiley and Sons Inc., New Jersey, 2003).

[2] R. W. Boyd, Nonlinear Optics (Elsevier Science, Amsterdam,2003).

[3] R. K. Chang, J. Ducuing, and N. Bloembergen, Dispersion of the Optical Nonlinearity in Semiconductoros, Phys. Rev. Lett. 15, 415 (1965).

[4] H. Zhong, Z. H. Levine, D. C. Allan, and J. W. Wilkins, Band-theoretic calculation of the optical activity tensor of $\alpha$-quartz and trigonal Se, Phys. Rev. B 48, 1384 (1993).

[5] J. L. P. Hughes and J. E. Sipe, Calculation of secondorder optical response in semiconductors, Phys. Rev. B 53, 10751 (1996).

[6] V. I. Gavrilenko and R. Q. Wu, Linear and nonlinear optical properties of group-III nitrides, Phys. Rev. B 61, 2632 (2000).

[7] D. J. Cai and G.-Y. Guo, Tuning linear and nonlinear optical properties of wurtzite GaN by c-axial stress, J. Phys. D: Appl. Phys. 42, 185107 (2009).

[8] M. J. Cheng, S. Q. Wu, Z.-Z. Zhu, and G.-Y. Guo, Large second-harmonic generation and linear electro-optic effect in trigonal selenium and tellurium, Phys. Rev. B 100, 035202 (2019).

[9] G. Y. Guo, K. C. Chu, D.-S. Wang, and C.-G. Duan, Linear and nonlinear optical properties of carbon nanotubes from first-principles calculations, Phys. Rev. B 69, 205416 (2004).

[10] G. Y. Guo and J. C. Lin, Second-harmonic generation and linear electro-optical coefficients of BN nanotubes, Phys. Rev. B 72, 075416 (2005); Erratum: Secondharmonic generation and linear electro-optical coefficients of BN nanotubes [Phys. Rev. B 72, 075416 (2005)], 77, 049901 (2008).

[11] M. Grüning and C. Attaccalite, Second harmonic generation in h-BN and $\mathrm{MoS}_{2}$ monolayers: Role of electron-hole interaction, Phys. Rev. B 89, 081102(R) (2014).

[12] M. L. Trolle, G. Seifert and T. G. Pedersen, Theory of excitonic second-harmonic generation in monolayer $\mathrm{MoS}_{2}$, Phys. Rev. B 89, 235410 (2014).

[13] C.-Y. Wang and G.-Y. Guo, Nonlinear optical properties of transition-metal dichalcogenide $\mathrm{MX}_{2}(\mathrm{M}=\mathrm{Mo}, \mathrm{W} ; \mathrm{X}=$
$\mathrm{S}, \mathrm{Se})$ monolayers and trilayers from first-principles calculations, J. Phys. Chem. C 119,13268 (2015).

[14] C. Attaccalite, A. Nguer, E. Cannuccia and M. Grüning, Strong second harmonic generation in $\mathrm{SiC}, \mathrm{ZnO}$ and $\mathrm{GaN}$ two-dimensional hexagonal crystalls from first-principles many-body calculations, Phys. Chem. Chem. Phys. 17, 9533 (2015).

[15] L. Hu, X. Huang, and D. Wei, Layer-independent and layer-dependent nonlinear optical properties of twodimensional GaX (X=S, Se, Te) nanosheets. Phys. Chem. Chem. Phys. 19,11131 (2017).

[16] H. Wang and X. F. Qian, Giant optical second harmonic generation in two-dimensional multiferroics. Nano Lett. 17, 5027 (2017).

[17] S. R. Panday, and B. M. Fregoso, Strong second harmonic generation in two-dimensional ferroelectric IVmonochalcogenides, J. Phys.: Condens. Matter 29, 43LT01 (2017); Corrigendum: Strong second harmonic generation in two-dimensional ferroelectric IVmonochalcogenides (2017 J. Phys.: Condens. Matter 29 43LT01), 30, 179501 (2018).

[18] C. Attaccalite, M. Palummo, E. Cannuccia and M. Grüning, Second-harmonic generation in single-layer monochalcogenides: A response from first-principles realtime similations, Phys. Rev. Mater. 3, 074003 (2019).

[19] Q. Wu and X.-C. Zhang, Ultrafast electro-optic field sensors, Appl. Phys. Lett. 68, 1604 (1996).

[20] A. G. Chynoweth, Surface Space-Charge Layers in Barium Titanate, Phys. Rev. 102, 705 (1956).

[21] A. M. Glass, D. von der Linde, and T. J. Negran, Highvoltage bulk photovoltaic effect and the photorefractive process in $\mathrm{LiNbO}_{3}$, Appl. Phys. Lett. 25, 233 (1974).

[22] S. M. Young and A. M. Rappe, First Principles Calculation of the Shift Current Photovoltaic Effect in Ferroelectrics, Phys. Rev. Lett. 109, 116601 (2012).

[23] A. Bhatnagar, A. R. Chaudhuri, Y. H. Kim, D. Hesse, and M. Alexe, Role of domain walls in the abnormal photovoltaic effect in $\mathrm{BiFeO}_{3}$, Nat. Commun. 4, 2835 (2013).

[24] J. A. Brehm, S. M. Young, F. Zheng, and A. M. Rappe, First-principles calculation of the bulk photovoltaic effect in the polar compounds $\mathrm{LiAsS}_{2}, \mathrm{LiAsSe}_{2}$, and $\mathrm{NaAsSe}_{2}$, J. Chem. Phys. 141, 204704 (2014). 
[25] T. Rangel, B. M. Fregoso, B. S. Mendoza, T. Morimoto, J. E. Moore, and J. B. Neaton, Large Bulk Photovoltaic Effect and Spontaneous Polarization of SingleLayer Monochalcogenides, Phys. Rev. Lett. 119, 067402 (2017).

[26] S.-J. Gong, F. Zheng, and A. M. Rappe, Phonon Influence on Bulk Photovoltaic Effect in the Ferroelectric Semiconductor GeTe, Phys. Rev. Lett. 121, 017402 (2018).

[27] L. A. Agapito, N. Kioussis, W. A. Goddard III, and N. P. Ong, Novel Family of Chiral-Based Topological Insulators: Elemental Tellurium under Strain, Phys. Rev. Lett. 110, 176401 (2013).

[28] H. Peng, N. Kioussis, and G. J. Snyder, Elemental tellurium as a chiral p-type thermoelectric material, Phys. Rev. B 89, 195206 (2014).

[29] M. Hirayama, R. Okugawa, S. Ishibashi, S. Murakami, and T. Miyake, Weyl Node and Spin Texture in Trigonal Tellurium and Selenium, Phys. Rev. Lett. 114, 206401 (2015).

[30] C. Şahin, J. Rou, J. Ma, and D. A. Pesin, PancharatnamBerry phase and kinetic magnetoelectric effect in trigonal tellurium, Phys. Rev. B 97, 205206 (2018).

[31] S. S. Tsirkin, P. A. Puente, and I. Souza, Gyrotropic effects in trigonal tellurium studied from first principles. Phys. Rev. B 97, 035158 (2018).

[32] H. O. H. Churchill, G. J. Salamo, S.-Q. Yu, T. Hironaka, X. Hu, J. Stacy, and I. Shih, Toward Single Atom Chains with Exfoliated Tellurium, Nanoscale Res. Lett. 12, 488 (2017).

[33] I. L. Li, J. P. Zhai, P. Launois, S. C. Ruan, and Z. K. Tang, Geometry, Phase Stability, and Electronic Properties of Isolated Selenium Chains Incorporated in a Nanoporous Matrix, J. Am. Chem. Soc. 127, 16111 (2005).

[34] D. J. Olechna and R. S. Knox, Energy-Band Structure of Selenium Chains, Phys. Rev. 140, A986 (1965).

[35] M. Springborg, and R. O. Jones, Sulfur and selenium helices: Structure and electronic properties, J. Chem. Phys. 88, 2652 (1988).

[36] P. Ghosh, M. U. Kahaly, and U. V. Waghmare, Atomic and electronic structures, elastic properties, and optical conductivity of bulk Te and Te nanowires: A firstprinciples study, Phys. Rev. B 75, 245437 (2007).

[37] M. U. Kahaly, P. Ghosh, S. Narasimhan, and U. V. Waghmare, Size dependence of structural, electronic, elastic, and optical properties of selenium nanowires: A first-principles study, J. Chem. Phys. 128, 044718 (2008).

[38] B. Tuttle, S. Alhassan, and S. Pantelides, Computational Predictions for Single Chain Chalcogenide-Based OneDimensional Materials, Nanomaterials (Basel) 7, 115 (2017).

[39] E. Andharia, T. P. Kaloni, G. J. Salamo, S.-Q. Yu, H. O. H. Churchill, and S. Barraza-Lopez, Exfoliation energy, quasiparticle band structure, and excitonic properties of selenium and tellurium atomic chains, Phys. Rev. B 98, 035420 (2018).

[40] Y. Y. Pan, S. Y. Gao, L. Yang, and J. Lu, Dependence of excited-state properties of tellurium on dimensionality: From bulk to two dimensions to one dimensions, Phys. Rev. B 98, 085135 (2018).

[41] J. K. Qin, G. Qiu, J. Jian, H. Zhou, L. M. Yang, A. Charnas, D. Y. Zemlyanov, C.-Y. Xu, X. F. Xu, W. Z. Wu, H. Y. Wang, and P. D. Ye, Controlled Growth of a Large-Size 2D Selenium Nanosheet and Its Electronic and Optoelectronic Applications, ACS Nano 11, 10222 (2017).

[42] Z. L. Zhu, X. L. Cai, S. H. Yi, J. L. Chen, Y. W. Dai, C. Y. Niu, Z. X. Guo, M. H. Xie, F. Liu, J.-H. Cho, Y. Jia, and Z. Y. Zhang, Multivalency-Driven Formation of Te-Based Monolayer Materials : A Combined FirstPrinciples and Experimental study, Phys. Rev. Lett. 119, 106101 (2017).

[43] L. Xian, A. P. Paz, E. Bianco, P. M Ajayan, and A. Rubio, Square selenene and tellurene: novel group VI elemental 2D materials with nontrivial topological properties, 2D Mater. 4, 041003 (2017).

[44] X.-X. Xue, Y.-X. Feng, L. Liao, Q.-J. Chen, D. Wang, L.-M. Tang, and K. Q. Chen, Strain tuning of electronic properties of various dimension elemental tellurium with broken screw symmetry, J. Phys.: Condens. Matter 30, 125001 (2018).

[45] S. Sharma, N. Singh, and U. Schwingenschlögl, TwoDimensional Tellurene as Excellent Thermoelectric Material, ACS Appl. Energy Mater. 1, 1950 (2018).

[46] T. T. Debela and H. S. Kang, Phase polymorphism and electronic structures of $\mathrm{TeSe}_{2}$, J. Mater. Chem. C 6, 10218 (2018).

[47] W. Zhang, Q. S. Wu, O. V. Yazyev, H. M. Weng, Z. X. Guo, W.-D. Cheng, and G.-L. Chai, Topological phase transitions driven by strain in monolayer tellurium, Phys. Rev. B 98, 115411 (2018).

[48] Y. Chen, J. Y. Liu, J. B. Yu, Y. G. Guo, and Q. Sun, Symmetry-breaking induced large piezoelectricity in Janus tellurene materials, Phys. Chem. Chem. Phys. 21, 1207 (2019).

[49] G. Liu, Z. B. Gao, and J. Ren, Anisotropic thermal expansion and thermodynamic properties of monolayer $\beta$ Te, Phys. Rev. B 99, 195436 (2019).

[50] J. L. Chen, Y. W. Dai, Y. Q. Ma, X. Q. Dai, W. K. Ho, and M. H. Xie, Ultrathin $\beta$-tellurium layers grown on highly oriented pyrolytic graphite by molecular-beam epitaxy, Nanoscale 9, 15945 (2017).

[51] Y. X. Wang, G. Qiu, R. X. Wang, S. Y. Huang, Q. X. Wang, Y. Y. Liu, Y. C. Du, W. A. Goddard III, M. J. Kim, X. F. Xu, P. D. Ye, and W. Z. Wu, Field-effect transistors made from solution-grown two-dimensional tellurene, Nat. Electron. 1, 228 (2018).

[52] J. Peng, Y. Pan, Z. Yu, J. J. Wu, J. C. Wu, Y. Zhou, Y. Q. Guo, X. J. Wu, C. Z. Wu, and Y. Xie, Two-Dimensional Tellurium Nanosheets Exhibiting an Anomalous Switchable Photoresponse with Thickness Dependence, Angew. Chem. Int. Ed. 57, 13533 (2018).

[53] A. Apte, E. Bianco, A. Krishnamoorthy, S. Yazdi, R. Rao, N. Glavin, H. Kumazoe, V. Varshney, A. Roy, F. Shimojo, E. Ringe, R. K Kalia, A. Nakano, C. S. Tiwary, P. Vashishta, V. Kochat, and P. M Ajayan, Polytypism in ultrathin tellurium, 2D Mater. 6, 015013 (2019).

[54] S. D. Kang, T. Dai, S. Dang, X. Y. Ma, G. L. Wang, H. W. Li, P. Hu, F. M. Yu, X. Zhou, S. X. Wu, S. W. Li, Broadband photoresponse of tellurium nanorods grown by molecular beam epitaxy, Chem. Phys. Lett. 729, 49 (2019).

[55] S. Deckoff-Jones, Y. X. Wang, H. T. Lin, W. Z. Wu, and J. J. Hu, Tellurene: A Multifunctional Material for Midinfrared Optoelectronics, ACS Photonics 6, 1632 (2019).

[56] X. C. Huang, J. Q. Guan, Z. J. Lin, B. Liu, S. Y. Xing, W. H. Wang, and J. D. Guo, Epitaxial Growth and Band 
Structure of Te Film on Graphene, Nano Lett. 17, 4619 (2017).

[57] J. S. Qiao, Y. H. Pan, F. Yang, C. Wang, Y. Chai, and W. Ji, Few-layer tellurium: one-dimensional-like layered elementary semiconductor with striking physical properties, Sci. Bull. 63, 159 (2018).

[58] Y. Wang, C. C. Xiao, M. G. Chen, C. Q. Hua, J. D. Zou, C. Wu, J. Z. Jiang, S. Y. A. Yang, Y. H. Lu, and W. Ji, Two-dimensional ferroelectricity and switchable spintextures in ultra-thin elemental Te multilayers, Mater. Horiz., 5, 521, (2018).

[59] C. Wang, X. Y. Zhou, J. S. Qiao, L. W. Zhou, X. H. Kong, Y. H. Pan, Z. H. Cheng, Y. Chai, and W. Ji, Charge-governed phase manipulation of few-layer tellurium, Nanoscale, 10, 22263, (2018).

[60] W. D. Teuchert, R. Geick, G. Landwehr, H. Wendel, and W.Weber, Lattice dynamics of trigonal selenium. I. Phonon spectra, J. Phys. C: Solid State Phys. 8, 3725 (1975).

[61] R. Keller, W. B. Holzapfel, and H. Schulz, Effect of pressure on the atom positions in Se and Te, Phys. Rev. B 16, 4404 (1977).

[62] P. E. Blöchl, Projector augmented-wave method, Phys. Rev. B 50, 17953 (1994).

[63] G. Kresse and J. Furthmüller, Efficient iterative schemes for ab initio total-energy calculations using a plane-wave basis set, Phys. Rev. B 54, 11169 (1996).

[64] G. Kresse and J. Furthmüller, Efficiency of ab-initio total energy calculations for metals and semiconductors using a plane-wave basis set, Comput. Mater. Sci. 6, 15 (1996).

[65] P. E. Blöchl, O. Jepsen, and O. K. Andersen, Improved tetrahedron method for Brillouin-zone integrations, Phys. Rev. B 49, 16223 (1994).

[66] J. W. Sun, A. Ruzsinszky, and J. P. Perdew, Strongly Constrained and Appropriately Normed Semilocal Density Functional, Phys. Rev. Lett. 115, 036402 (2015).

[67] H. W. Peng, Z.-H. Yang, J. P. Perdew, and J. W. Sun, Versatile van der Waals Density Functional Based on a Meta-Generalized Gradient Approximation, Phys. Rev. X 6, 041005 (2016).

[68] T. Thonhauser, V. R. Cooper, S. Li, A. Puzder, P. Hyldgaard, and D. C. Langreth, Van der Waals density functional: Self-consistent potential and the nature of the van der Waals bond, Phys. Rev. B 76, 125112 (2007).

[69] J. P. Perdew, K. Burke, and M. Ernzerhof, Generalized Gradient Approximation Made Simple, Phys. Rev. Lett. 77, 3865 (1996).

[70] S. Grimme, Semiempirical GGA-Type Density Functional Constructed with a Long-Range Dispersion Correction, J. Comput. Chem. 27, 1787 (2006).

[71] G. Y. Guo and J. C. Lin, Systematic ab initio study of the optical properties of BN nanotubes, Phys. Rev. B 71, 165402 (2005).

[72] B. Adolph, J. Furthmüller, and F. Bechstedt, Optical properties of semiconductors using projector-augmented waves, Phys. Rev. B 63, 125108 (2001).

[73] L. Prussel and V. Véniard, Linear electro-optic effect in semiconductors: $A b$ initio description of the electronic contribution, Phys. Rev. B 97, 205201 (2018).

[74] J. E. Sipe and A. I. Shkrebtii, Second-order optical response in semiconductors, Phys. Rev. B 61, 5337 (2000).
[75] T. Cheiwchanchamnangij and W. R. L. Lambrecht, Quasiparticle band structure calculation of monolayer, bilayer, and bulk MoS 2 , Phys. Rev.B 85, 205302 (2012).

[76] S. Tutihasi and I. Chen, Optical properties and band structure of trigonal selenium, Phys. Rev. 158, 623 (1967).

[77] V. B. Anzin, M. I. Eremets, Y. V. Kosichkin, A. I. Nadezhdinskii and A. M. Shirokov, Measurement of the energy gap in tellurium under pressure, Phys. Status Solidi A 42, 385 (1977).

[78] J. Heyd, G. E. Scuseria, and M. Ernzerhof, Hybrid functionals based on a screened Coulomb potential, J. Chem. Phys. 118, 8207 (2003).

[79] Z. H. Levine and D. C. Allan, Quasiparticle calculation of the dielectric response of silicon and germanium, Phys. Rev. B 43, 4187 (1991).

[80] T.-Y. Cai, S.-C. Liu, S. Ju, C.-Y. Liu and G.-Y. Guo, Multiferroic double perovskites $\mathrm{ScFe}_{1-x} \mathrm{Cr}_{x} \mathrm{O}_{3}(1 / 6 \leq$ $x \leq 5 / 6)$ for highly efficient photovoltaics and spintronics, Phys. Rev. Appl. 8, 034034 (2017).

[81] F. Hüser, T. Olsen and K. S. Thygesen, Quasiparticle GW calculations for solids, molecules, and twodimensional materials, Phys. Rev. B 87, 235132 (2013).

[82] M. M. Ugeda, A. J. Bradley, S.-F. Shi, F. H. da Jornada, Y. Zhang, D. Y. Qiu, W. Ruan, S.-K. Mo, Z. Hussain, Z.-X. Shen, F. Wang, S. G. Louie and M. F. Crommie, Giant bandgap renormalization and excitonic effects in a monolayer transition metal dichalcogenide semiconductor, Nature Mater. 13, 1091 (2014).

[83] F. A. Rasmussen and K. S. Thygesen, Computational 2D materials database: Electronic structure of transitionmetal dichalcogenides and oxides, J. Phys. Chem. C 119, 13169 (2015).

[84] Y. M. Fang, S. Q. Wu, Z.-Z. Zhu, and G.-Y. Guo, Large magneto-optical effects and magnetic anisotropy energy in two-dimensional $\mathrm{Cr}_{2} \mathrm{Ge}_{2} \mathrm{Te}_{6}$, Phys. Rev. B 98, 125416 (2018).

[85] See Supplemental Material at http://link.aps.org/supplemental/ for Figs. S1-S4.

[86] X. X. Jiang, S. G. Zhao, Z. S. Lin, J. H. Luo, P. D. Bristowe, X. G. Guan, and C. T. Chen, The role of dipole moment in determining the nonlinear optical behavior of materials: ab initio studies on quaternary molybdenum tellurite crystals, J. Mater. Chem. C 2, 530 (2014).

[87] A. Cammarata, W. G. Zhang, P. S. Halasyamani, and J. M. Rondinelli, Microscopic Origins of Optical Second Harmonic Generation in Noncentrosymmetric-Nonpolar Materials, Chem. Mater. 26, 5773 (2014).

[88] J. Ingers, K. Maschke, and S. Proennecke, Opticaltransition-matrix elements between localized electronic states in disordered one-dimensional systems, Phys. Rev. B 37, 6105 (1988).

[89] J.-H. Song, A. J. Freeman, T. K. Bera, I. Chung, and M. G. Kanatzidis, First-principles prediction of an enhanced optical second-harmonic susceptibility of lowdimensional alkali-metal chalcogenides, Phys. Rev. B 79, 245203 (2009). 